\title{
Solar wind and substorm excitation of the wavy current sheet
}

\author{
C. Forsyth ${ }^{1,2}$, M. Lester ${ }^{1}$, R. C. Fear ${ }^{1}$, E. Lucek ${ }^{3}$, I. Dandouras ${ }^{4}$, A. N. Fazakerley ${ }^{2}$, H. Singer ${ }^{5}$, and T. K. Yeoman ${ }^{1}$ \\ ${ }^{1}$ Dept. Physics and Astronomy, University of Leicester, Leicester, LE1 7RH, UK \\ ${ }^{2}$ Mullard Space Science Laboratory, University College London, Holmbury St. Mary, Dorking, Surrey, RH5 6NT, UK \\ ${ }^{3}$ Blackett Laboratory, Imperial College, London, SW7 2BZ, UK \\ ${ }^{4}$ CESR/CNRS-Université de Toulouse, 9 Avenue du Colonel Roche, B.P. 4346, 31028, Toulouse Cedex 4, France \\ ${ }^{5}$ National Oceanic and Atmospheric Administration, Boulder, CO 80305, USA
}

Received: 5 August 2008 - Revised: 8 May 2009 - Accepted: 1 June 2009 - Published: 19 June 2009

\begin{abstract}
Following a solar wind pressure pulse on 3 August 2001, GOES 8, GOES 10, Cluster and Polar observed dipolarizations of the magnetic field, accompanied by an eastward expansion of the aurora observed by IMAGE, indicating the occurrence of two substorms. Prior to the first substorm, the motion of the plasma sheet with respect to Cluster was in the $Z_{\mathrm{GSM}}$ direction. Observations following the substorms show the occurrence of current sheet waves moving predominantly in the $-Y_{\mathrm{GSM}}$ direction. Following the second substorm, the current sheet waves caused multiple current sheet crossings of the Cluster spacecraft, previously studied by Zhang et al. (2002). We further this study to show that the velocity of the current sheet waves was similar to the expansion velocity of the substorm aurora and the expansion of the dipolarization regions in the magnetotail. Furthermore, we compare these results with the current sheet wave models of Golovchanskaya and Maltsev (2005) and Erkaev et al. (2008). We find that the Erkaev et al. (2008) model gives the best fit to the observations.
\end{abstract}

Keywords. Magnetospheric physics (Magnetotail; Solar wind-magnetosphere interactions; Storms and substorms)

\section{Introduction}

The magnetotail current sheet has been extensively studied since spacecraft technology allowed for in-situ measurements to be made. These in-situ measurements have shown that the current sheet often has a flapping or wavy motion (e.g. Sergeev et al., 2004, 2006) which is not only an interesting dynamical feature, warranting its own investigation (e.g. Zhang et al., 2002), but also facilitates the further investiga-

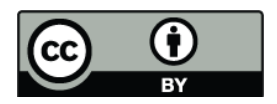

Correspondence to: C. Forsyth (cfo@mssl.ucl.ac.uk) tion of the current sheet structure (e.g. Sergeev et al., 2003; Runov et al., 2006).

Early studies of the current sheet motion were restricted to single spacecraft observations and as such, the motion of current sheet waves could not be determined unambiguously. It was suggested that the current sheet motion may be due to variations in the solar wind (Speiser and Ness, 1967) or waves propagating across the tail (Nakagawa and Nishida, 1989). Observations made by the multi-spacecraft Cluster mission have removed this ambiguity. Case studies (Zhang et al., 2002; Runov et al., 2003; Sergeev et al., 2003; Zhang et al., 2005) and statistical studies (Sergeev et al., 2006) have shown that during periods in which the Cluster spacecraft crossed the current sheet several times, the current sheet tended to be highly tilted in the $Y Z$ plane and exhibited transient features in the dusk/dawn direction away from the centre of the tail. Zhang et al. (2005) also showed that current sheet waves extended over $\sim 10 R_{E}$ in the Earthwards direction by comparing data from the Cluster and Double Star missions. Using Cluster data to investigate the validity of the minimum variance analysis technique (MVA; Sonnerup and Cahill, 1967; Sonnerup and Scheible, 1998), Sergeev et al. (2006) used Geotail data to show that the occurrence rate of plasma sheet flapping with radial and cross tail location was comparable to the occurrence rate of bursty bulk flows (BBFs). They also showed that superposed epoch analysis of the $A E$ index at the time of plasma sheet flapping suggested that plasma sheet flapping was likely during substorm expansion phases. This was in agreement with previous studies (e.g. Bauer et al., 1995; Sergeev et al., 1998) that suggested that substorms might cause current sheet waves.

Prior to substorm expansion phase onset, the magnetotail becomes stretched and less dipolar (e.g. Fairfield and Ness, 1970; Kokubun and McPherron, 1981; Nagai, 1982). Following the expansion phase onset, the tail magnetic field topology becomes more dipolar, especially at geosynchronous orbit, coupled with the diversion of the cross tail

Published by Copernicus Publications on behalf of the European Geosciences Union. 

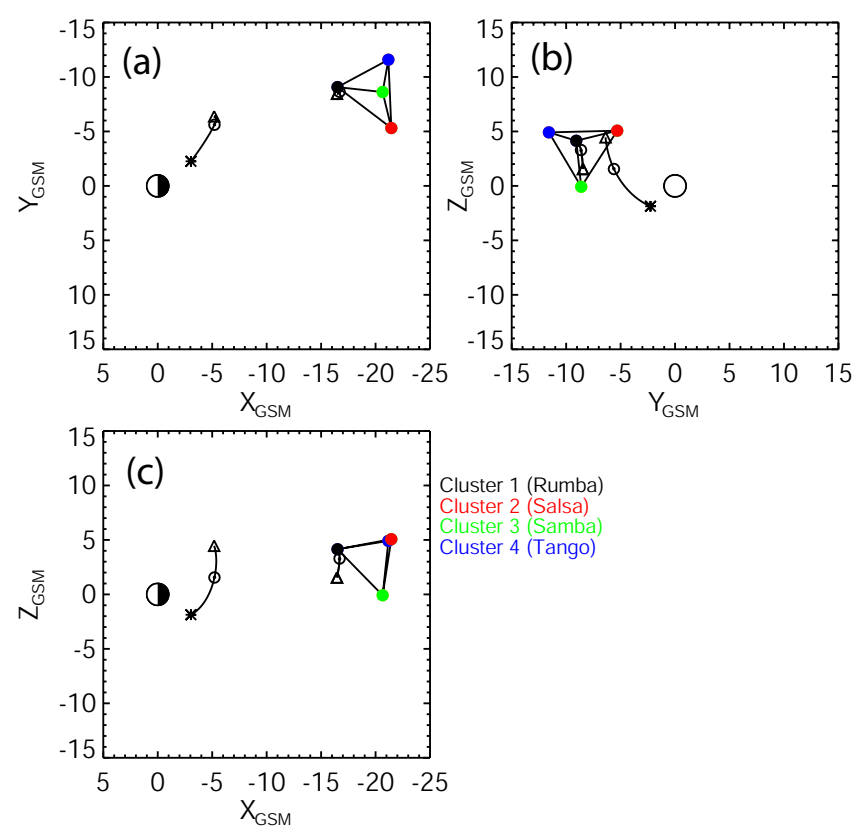

Fig. 1. Plots of the Cluster and Polar orbital positions between 06:00 and 12:00 UT in the (a) $X Y$, (b) $Y Z$ and (c) $X Z$ in GSM coordinates. The Cluster tetrahedron at 06:00 UT is magnified by a factor of 20. Cluster 1 (Rumba, black circle) is plotted at the correct location. The locations of Cluster 1 and Polar at 09:00 and 12:00 UT are shown by open circles and triangles respectively.

current into the ionosphere via the substorm current wedge (McPherron et al., 1973; Rostoker, 1974; Nagai, 1982). This dipolarization of the field is initially localised and then expands azimuthally (Kokubun and McPherron, 1981; Nagai, 1982). This dipolarization can extend over several $R_{E}$ downtail (Nakamura et al., 2005) and the azimuthal propagation of the dipolarization at geosynchronous orbit has been associated with the azimuthal expansion of the substorm auroral bulge (Liou et al., 2002). Furthermore, it has been shown that the expansive motion of the auroral bulge is determined by the polarity of the IMF $B_{Y}$ component if the IMF $B_{Y}$ is fairly steady, although the speed of the expansion is only moderately correlated with the magnitude of the IMF $B_{Y}$ (Liou et al., 2006). Liou and Ruohoniemi (2006a,b) used two case studies to show that the direction of the expansion of the auroral bulge was dependent on the plasma convection flows at the location of the auroral breakup.

This study investigates the tail and auroral dynamics following a solar wind pressure pulse and two substorms. We expand upon the study by Zhang et al. (2002), including data from Polar, GOES 8 and 10 and Cluster, enabling us to determine a global picture of the magnetotail dynamics at this time. Comparing magnetotail data and auroral observations, we show that the azimuthal expansion of dipolarized regions extending radially across $12 R_{E}$ of the magnetotail is related to an auroral expansion. Using four-spacecraft timing analysis, we show that the expansion velocity of these regions is similar to the motion of the current sheet waves following them. Furthermore, we test two models of current sheet waves in order to determine which is more likely for this event. From these observations and theoretical models, we speculate that the velocities of both the waves and the dipolarization fronts are controlled by the configuration of the magnetotail.

\section{Instrumentation}

In this study, data are employed from the Cluster FluxGate Magnetometers (FGM; Balogh et al., 2001), Cluster Ion Spectrometer CODIF sensors (CIS-CODIF; Rème et al., 2001), Cluster Plasma Electron and Current Experiment HEEA sensors (PEACE-HEEA; Johnstone et al., 1997), Polar Magnetic Field Experiment (MFE; Russell et al., 1995) and GOES 8 and 10 Magnetometers. We note that the GOES 8 data are subject to an offset in the $B_{Z}$ component and, hence, apply a correction of $-7 \mathrm{nT}$ (Tsyganenko et al., 2003). Prior to 08:08 UT, the FGM onboard Cluster was running in EXT mode. This mode stores spin resolution FGM data in burst mode memory at times when the spacecraft are not telemetering data. The data are telemetered to the ground at the first opportunity. These data are calibrated using the onboard calibrations and have an increased uncertainty in the time-stamps due to the collection method (see Balogh et al., 2001, for details). After 08:08 UT, FGM data based on the $5 \mathrm{~Hz}$ resolution data from the Cluster Active Archive (CAA) are employed. These data have been calibrated to facilitate the multi-spacecraft analysis techniques used in this study. Polar MFE data have a temporal resolution of $6 \mathrm{~s}$, whereas GOES MAG data have a temporal resolution of $60 \mathrm{~s}$. The moments from the PEACE-HEEA sensors have been calculated on the ground from a reduced angular resolution 3D particle distribution (3DX - see Johnstone et al., 1997). Ground moments use improved calibrations and improved corrections for the effects of the spacecraft potential. The 3-D distributions are not telemetered on every spin, so the moments have a lower cadence which varies between spacecraft. Spacecraft data are presented in GSM coordinates unless otherwise stated.

Figure 1 shows the location of the Cluster and Polar spacecraft and Fig. 2 shows that of Polar, GOES 8 and GOES 10 in GSM coordinates between 06:00 and 12:00 UT. The Cluster tetrahedron at 06:00 UT is magnified by a factor of 20 , with Cluster 1 plotted at the correct location. The locations of Polar, GOES 8 and GOES 10 at 06:00, 09:00 and 12:00 UT are shown as asterisks, open circles and triangles, respectively. The location of Cluster 1 at 09:00 and 12:00 UT is shown as an open circle and triangle respectively. Cluster was in the northern magnetosphere, with Cluster 1 at $[-16.6,-9.1,4.2] R_{E}$ at 06:00 UT and moved to 

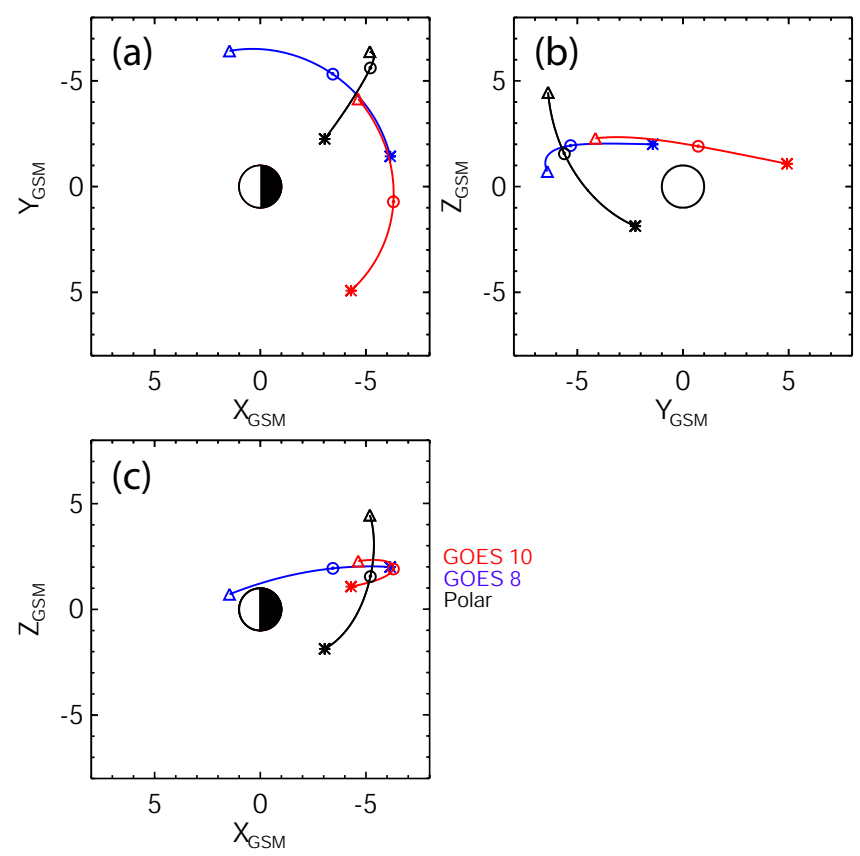

Fig. 2. Plots of the GOES 8 (blue), GOES 10 (red) and Polar (black) orbital positions between 06:00 and 12:00 UT in the (a) $X Y$, (b) $Y Z$ and (c) $X Z$ in GSM coordinates. The locations of the spacecraft at 06:00, 09:00 and 12:00 UT are represented by asterisks, open circles and triangles, respectively.

[-16.6,-8.5,1.5] $R_{E}$ by $12: 00 \mathrm{UT}$. Cluster 1 was the most Earthward spacecraft, whereas Cluster 2, 3 and 4 were at approximately the same downtail distance. Cluster 2 (red) was the most duskward and northernmost spacecraft. Cluster 3 (green) was the southernmost spacecraft and Cluster 4 (blue) was closest to dawn. The spacecraft were separated by $1700-2000 \mathrm{~km}$. Polar was initially in the southern magnetosphere at [-3.0,-2.3,-1.9] $R_{E}$ at 06:00 UT moving to $[-5.2,-6.4,4.5] R_{E}$ by $12: 00$ UT. The GOES spacecraft were in the northern magnetosphere throughout the interval. GOES 8 (blue) was the most dawnward spacecraft, initially at $[-6.1,-1.4,2] R_{E}$ and moved to $[1.5,-6.4,0.7] R_{E}$ by 12:00 UT, passing through the magnetic local time sector of Cluster and Polar. The closest separation of Polar and GOES 8 occurred at 08:29 UT, with the spacecraft passing within $9600 \mathrm{~km}$ of each other. GOES 10 (red) was initially located in the pre-midnight sector at $[-4.3,4.9,1.1] R_{E}$, moving to $[-4.6,-4.1,2.3] R_{E}$ by 12:00 UT.

Figure 3 shows the magnetic footprints of Cluster 1, Polar, GOES 8 and GOES 10 between 06:00 and 12:00 UT, calculated using the Tsyganenko (1989) model (hereafter referred to as the T89 model) with an input of $K_{P}=4$, in MLT - invariant latitude coordinates from the Altitude Adjusted Corrected GeoMagnetic coordinate system (Baker and Wing, 1989). Cluster remained close to 02:00 MLT throughout, whereas Polar was located at $3 \pm 0.5$ MLT throughout.

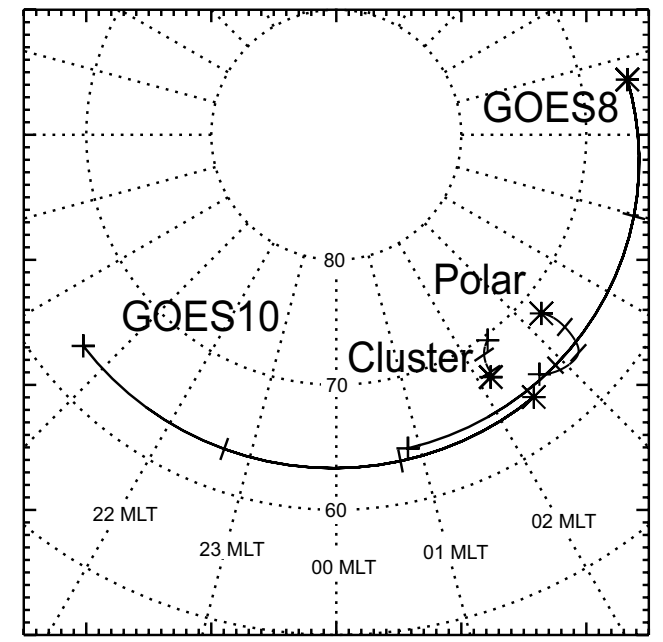

Fig. 3. MLT - invariant latitude (AACGM) locations of the magnetic footprints of Cluster 1, Polar, GOES 8 and GOES 10 between 06:00 and 12:00 UT calculated using the T89 model. The initial and final positions of the spacecraft are shown as crosses and asterisks respectively. The spacecraft positions are marked by dashes at 2-hourly intervals.

From 07:30 to 08:30 UT GOES 8, Cluster and Polar were within one hour of MLT of each other.

Solar wind data are provided by the magnetometer (Smith et al., 1998) and Solar Wind Electron Proton Alpha Monitor (SWEPAM; McComas et al., 1998) instruments on board the ACE spacecraft (Stone et al., 1998). During the interval ACE was situated at approximately $[245,-15,30] R_{E}$, sunward of the Earth. Solar wind data from ACE are lagged by comparing the IMF with magnetometer data from the IMAGE magnetometer array (Luhr, 1994; Viljanen and Häkkinen, 1997), which was on the dayside between 07:00 and 13:00 MLT, following the technique demonstrated by Volwerk et al. (2004).

Northern Hemisphere auroral data are also employed from the Far UltraViolet imager Wideband Imaging Camera (FUV-WIC; Mende et al., 2000a,b,c) on board the IMAGE spacecraft. During the interval, IMAGE was passing over the northern polar region, passing through apogee at 08:10 UT, with FUV-WIC imaging the northern polar region.

\section{Observations}

\subsection{Interval overview and solar wind conditions}

Solar wind ram pressure, density and velocity data from ACE are shown in Fig. 4 in panels $(\mathrm{a}-\mathrm{c})$, respectively. The IMF $B_{X}, B_{Y}$ and $B_{Z}$ are shown in panels (d-f) in GSM coordinates. The IMF clock angle is shown in panel $(\mathrm{g})$. The interval covered is 06:00-12:00 UT. In order to determine the solar wind conditions at Earth, it is necessary to lag the 


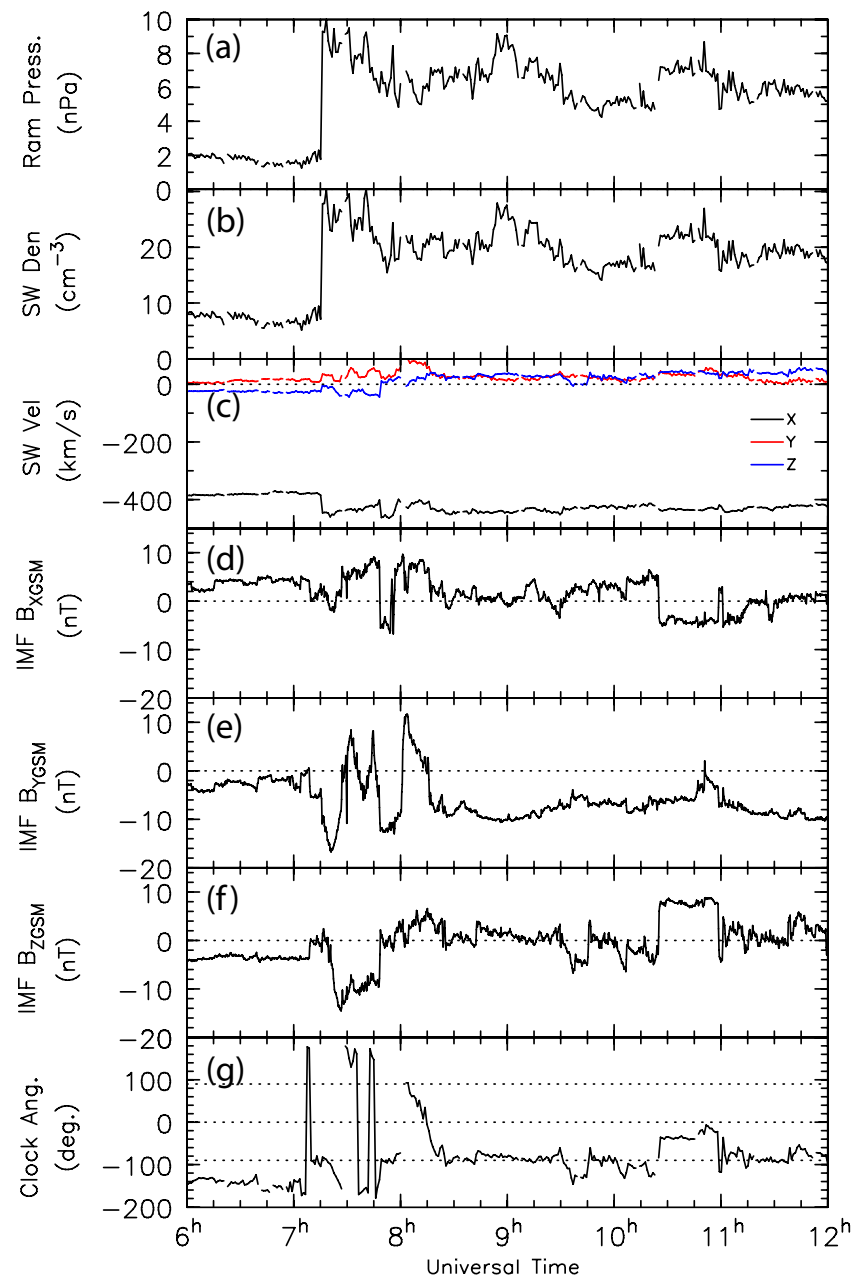

Fig. 4. Stack plot of solar wind and IMF data from ACE. The data have been lagged by $50 \mathrm{~min}$. Panel (a) shows the solar wind ram pressure. Panel (b) shows the ion density. Panel (c) shows the ion velocities in the $X$ (black), $Y$ (red) and $Z$ (blue) directions. Panels (d-f) show the IMF $B_{X}, B_{Y}$, and $B_{Z}$ in GSM coordinates respectively. Panel (g) shows the IMF clock angle in degrees.

data from ACE. It has previously been shown that the highlatitude ionosphere and the IMF are strongly coupled, especially following solar wind pressure pulses (e.g. Volwerk et al., 2004). The northward $(H)$ component of the magnetic field from a number of the IMAGE magnetometer chain magnetometers, which cover 58-76 ${ }^{\circ}$ MLAT and were on the dayside during the interval, are shown in Fig. 5. The data from the selected stations is shown in order of descending magnetic latitude. At 07:15 UT there was a distinct change in the magnetic field. Stations north of Bear Island (BJN) showed large, long period variations, whereas stations south of Masi (MAS) showed a lower amplitude variability on a much smaller timescale. Comparing the magnetometer positions with images from FUV-WIC on the IMAGE spacecraft (not shown) indicates that this difference in the dayside

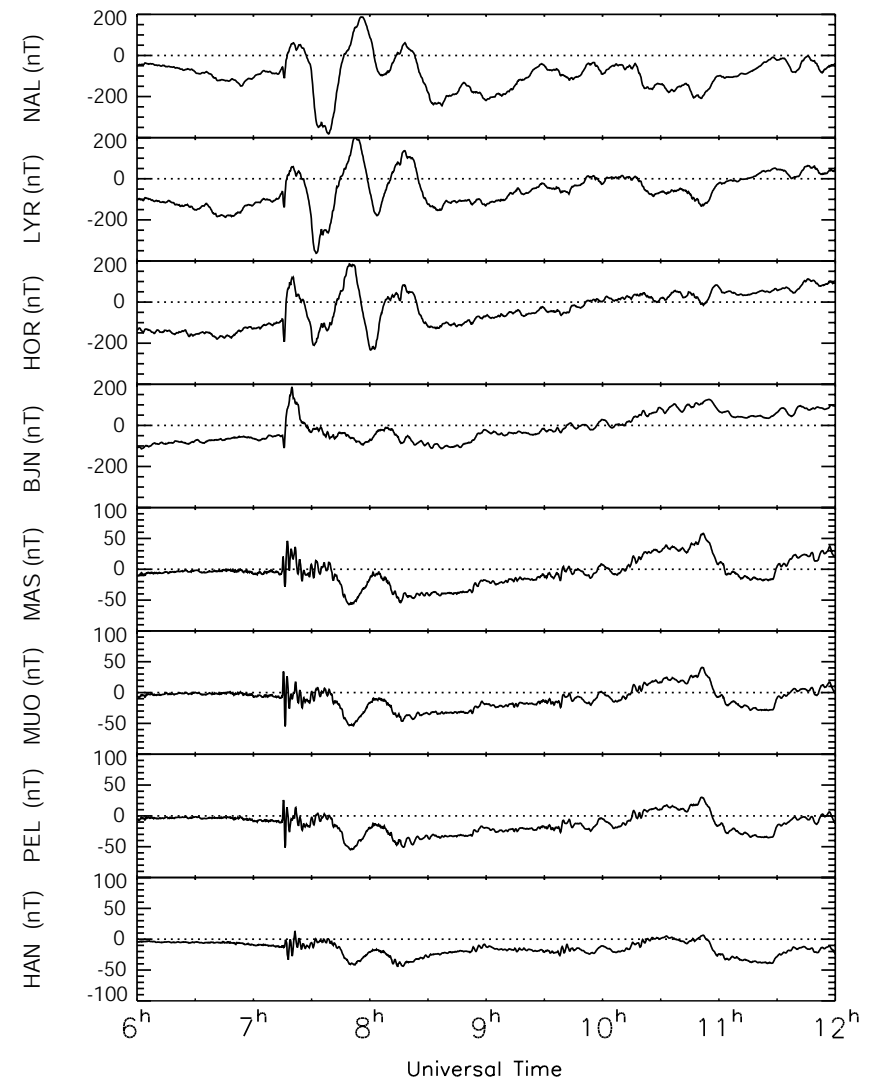

Fig. 5. Stack plot of $H$ component ground magnetometer data from selected stations in the IMAGE network. The dotted horizontal lines represent $0 \mathrm{nT}$.

magnetic field response to the pressure pulse was dependent on whether stations were on open or closed field-lines. The increased variability of the dayside magnetic field suggests that the lag between ACE and the Earth was 50 min. Data from ACE are in Fig. 4 are presented with this lag applied. This is less than, but comparable with, the $60 \mathrm{~min}$ lag used by Zhang et al. (2002), and the $64 \mathrm{~min}$ lag predicted by the model of Khan and Cowley (1999).

At 07:15 UT, a solar wind pressure pulse, in which the solar wind ram pressure increased by a factor of five dominated by a change in the solar wind density, impacted the magnetosphere (Fig. $4 \mathrm{a}-\mathrm{c}$ ). The ram pressure then slowly decreased, reaching $6 \mathrm{nPa}$ by the end of the interval, with increases of $\sim 2 \mathrm{nPa}$ at 08:55 and 10:20 UT. Prior to the initial pressure pulse, the ram pressure was $2 \mathrm{nPa}$ and the IMF was consistently southward. Data from 00:00 UT (not shown) indicates that the IMF was southward for almost $5 \mathrm{~h}$ prior to the interval shown in Fig. 4. Just before the pressure pulse, the IMF $B_{Z}$ dropped to $0 \mathrm{nT}$. Coincident with the pressure pulse, the IMF $B_{Z}$ magnitude increased briefly before decreasing to vary about $0 \mathrm{nT}$. The IMF turned northward at 07:48 UT and varied about northward and southward from that time. Also 


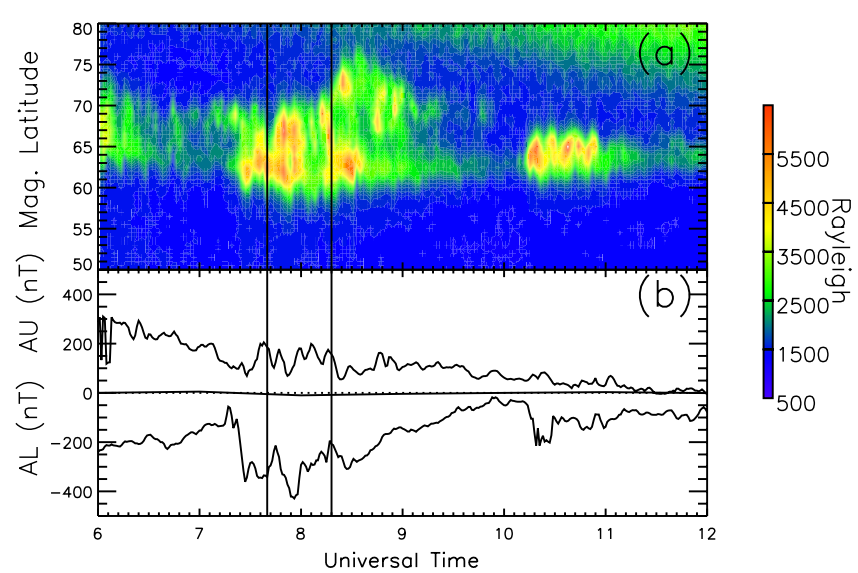

Fig. 6. (a) Keogram of auroral intensity along the 00:00 MLT meridian between $50^{\circ}$ and $80^{\circ}$ magnetic latitude from 06:00 to 12:00 UT. (b) The $A U$ and $A L$ auroral indices between 06:00 and 12:00 UT. The vertical lines indicate substorm onsets.

following the pressure pulse, $B_{Y}$ varied pseudo-periodically about $0 \mathrm{nT}$ with a periodicity of 20-30 min until 08:20 UT, at which time it became persistently negative at $-8 \mathrm{nT}$.

Figure 6 shows (a) a keogram of auroral intensity along the midnight meridian from the FUV-WIC instrument onboard the IMAGE spacecraft and (b) the $A L$ and $A U$ auroral indices between 06:00 and 12:00 UT. Prior to the solar wind pressure pulse, the auroral oval was dim and covered $65^{\circ}$ to $70^{\circ}$ magnetic latitude. Shortly after the arrival of the solar wind pressure pulse, the auroral intensity increased, with a large increase at the equatorward edge of the auroral oval. At 07:40UT, the bright aurora expanded rapidly polewards. A further poleward expansion of the aurora was observed at 08:18 UT. These auroral expansions were accompanied by decreases in the $A L$ index, indicating the occurrence of two substorm expansion phases at these times. These are indicated as black lines in Fig. 6. After 09:00 UT, the aurora along the midnight meridian dimmed to levels similar to those prior to the pressure pulse and retreated equatorwards. There was a further enhancement and poleward expansion between 10:15 and 11:00 UT. The $A U$ index showed an almost steady decrease throughout the interval, with slight enhancements between 07:30 and 08:30 UT.

\subsection{Magnetospheric compression and magnetotail dipo- larizations}

Figure 7 shows a stack plot of magnetometer data from GOES 8, 10 and Polar. Data from GOES 8 is represented by the blue line, data from GOES 10 is represented by the red line and data from Polar is represented by the black line in each panel. The T89 magnetic field model is shown as the dashed lines in the respective colour of the spacecraft. Panels (a-c) show the $B_{X}, B_{Y}$ and $B_{Z}$ components of the mag-

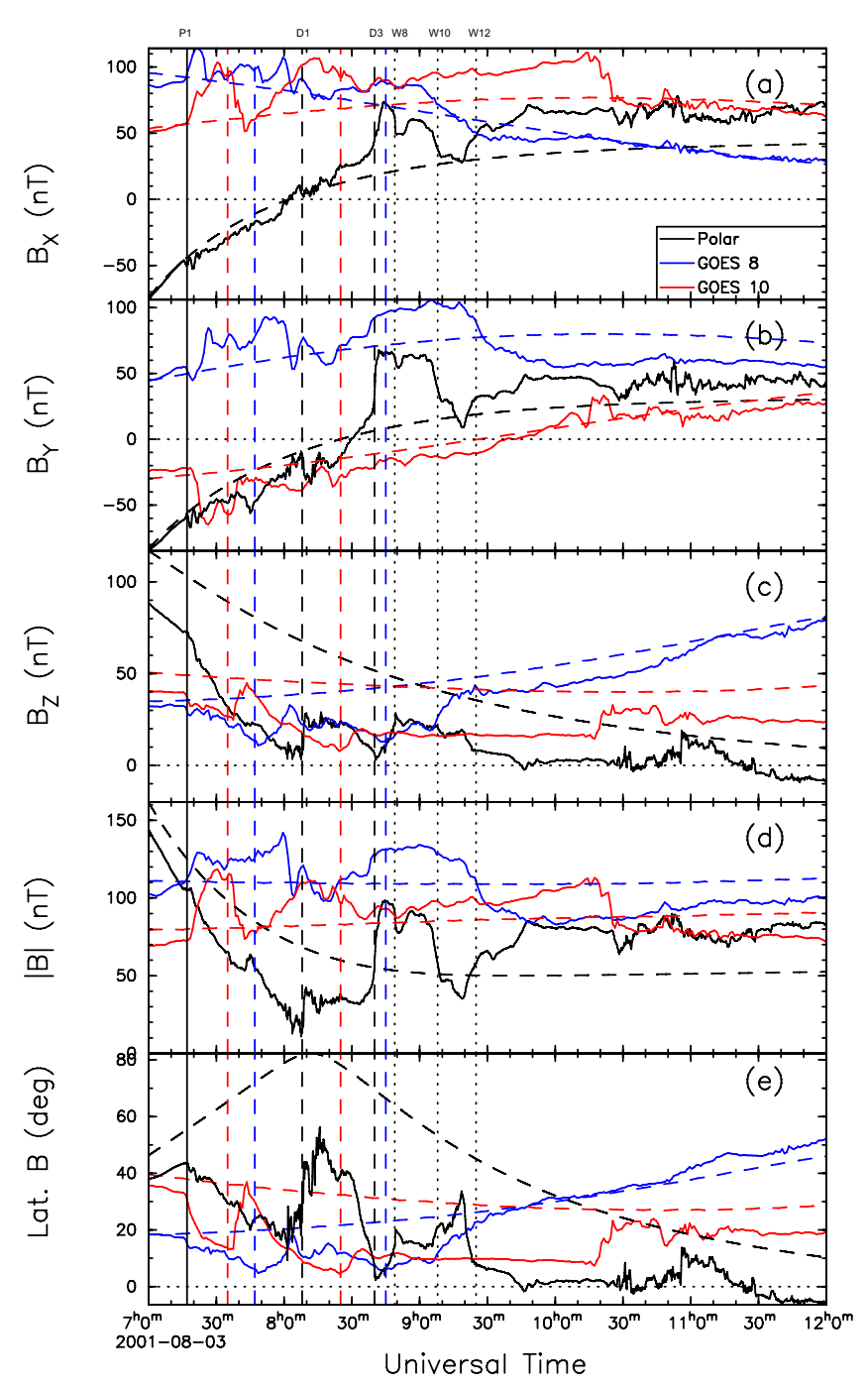

Fig. 7. Stack plot of magnetometer data from GOES 8 (blue) and GOES 10 (red) and Polar (black). Panels (a-c) show the $B_{X}, B_{Y}$ and $B_{Z}$ magnetic field components in GSM coordinates. Panel (d) shows the magnetic field magnitude. Panel (e) shows the angle between the $B_{Z}$ component and the component of the magnetic field in the $X Y$ plane (latitude angle). The dashed traces show the output from the T89 magnetic field model in the respective colours of the spacecraft. The horizontal dotted lines indicate zero in each panel. The dashed vertical lines indicate the times of the dipolarizations and the dotted vertical lines indicate the current sheet wave as observed by the various spacecraft and shown in their respective colours. The solid vertical line indicates the effects of the pressure pulse.

netic field in GSM coordinates. Panel (d) shows the magnetic field magnitude. Panel (e) shows the magnetic field latitude angle, taken to be the angle between the $B_{Z}$ component and the magnetic field component in the $X Y$ plane.

Around 07:00 UT, the magnetic fields at the GOES spacecraft were reasonably well matched by the T89 model, 


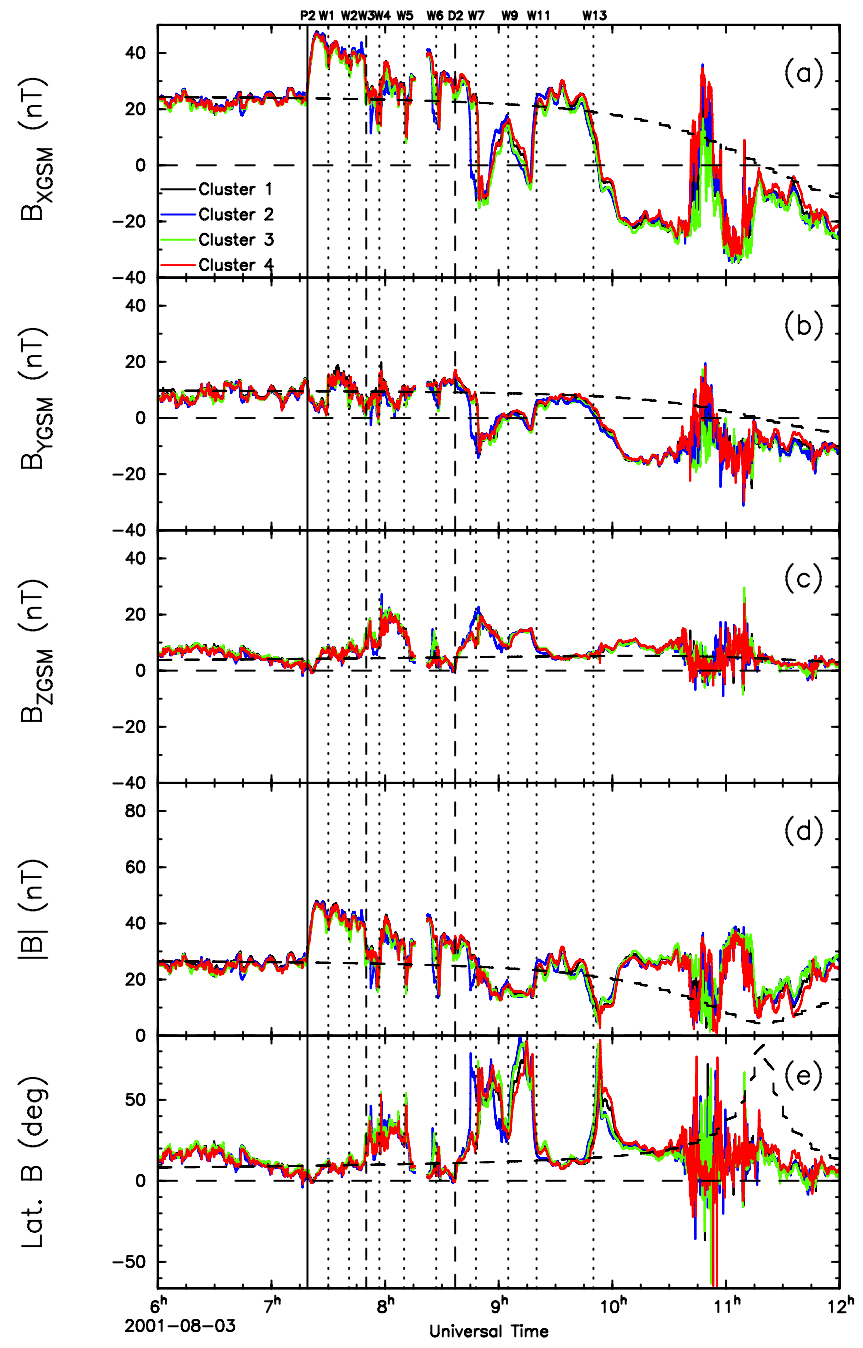

Fig. 8. Plot of FGM data Cluster. Panels (a-c) show the $B_{X}, B_{Y}$ and $B_{Z}$ magnetic field components in GSM coordinates. Panel (d) shows the magnetic field magnitude. Panel (e) shows the magnetic field latitude angle. The black dashed traces are the model field components from the T89 model based on the centre of the Cluster tetrahedron. The solid vertical line indicates the effects of the solar wind pressure pulse. The dotted lines indicate the current sheet waves. The dashed lines indicate the times of the dipolarizations of the field.

although, compared to the model, the magnetic field was slightly stretched, indicated by the slightly depressed $B_{Z}$ components (Fig. 7c), particularly at GOES 10. Data from the Polar spacecraft shows that it also detected a stretched field at this time in the post-midnight sector (Fig. 7c). At 07:15 UT, $B_{X}$ and $B_{Y}$ increased in magnitude at GOES 8 followed shortly by a larger increase in $B_{X}$ and $B_{Y}$ at GOES 10 . Since these increases are not associated with dipolarizations of the field, this indicates that the spacecraft detected an increase in the Region 1 currents caused by the pressure pulse.
Polar detected a circularly polarized wave at this time (labelled P1) with a period of $\sim 150 \mathrm{~s}$. GOES 10 detected two dipolarizations of the magnetic field at 07:35 and 08:25 UT and GOES 8 detected two dipolarizations at 07:47 UT and 08:45 UT, indicated by the dashed lines in Fig. 7. A final dipolarization of the magnetic field at GOES 8 at 09:10 UT brought the magnetic field level back towards the model field level, suggesting that the spacecraft moved out of the disturbed tail region and round onto the flanks of the magnetosphere. Polar detected two dipolarizations of the magnetic field at 08:08 and 08:40 UT respectively, labelled (D1 and D3). The first of these dipolarizations was very rapid, with the $B_{Z}$ component of the magnetic field increasing by $20 \mathrm{nT}$ in $45 \mathrm{~s}$. This was in contrast to the dipolarizations at the GOES spacecraft, which occurred over approximately $5 \mathrm{~min}$. The second dipolarization was associated with a large increase in $B_{X}$ and $B_{Y}$, with similar but more extended increases observed by GOES 8 . This indicates that GOES 8 and Polar detected the Earthward directed current that makes up part of the substorm current wedge. It is interesting to note that GOES 10 did not see a signature of the return current, although this is likely due to the location of the spacecraft at that time. The dipolarization at GOES 10 at 10:15 UT was due to the substorm expansion phase observed in the AL indices and auroral keogram (Fig. 6).

Figure 8 is a stack plot of magnetic field data from Cluster in the same format as Fig. 7. Figure 9 shows the particle moments from the Cluster CIS and PEACE instruments. Data from CIS were only available from Cluster 1 and 4. Panels (a) and (b) show the ion temperature and density. Panels (c-e) show the $X, Y$ and $Z$ components of the ion velocity. Panels ( $\mathrm{f}-\mathrm{j}$ ) show similar data for the electrons. The dashed lines show the times of the dipolarizations and the dotted lines indicate the current sheet waves.

From 06:45 UT, $B_{Z}$ at Cluster dropped steadily reaching half the model value by 07:15 UT, indicating that the magnetotail was stretching. At 07:18 UT, $B_{X}$ and the magnetic field magnitude rapidly doubled (labelled P2), accompanied by a drop in the latitude angle, indicating that Cluster detected the compression of the magnetotail by the solar wind pressure pulse. Following the pressure pulse, there were two large dipolarizations of the field starting at 07:50 and 08:37 UT observed as a more than doubling of $B_{Z}$ (Fig. 8c) and indicated by the vertical dashed lines in the plot. The first of these is associated with a drop in the $B_{X}$ component, indicating that it occurred when Cluster first detected a current sheet wave and is discussed in the next section. The second of these (labelled D2), for which ion moments were available, was accompanied by a slight increase in the ion temperature to $95 \mathrm{MK}$ at Cluster 1 and 4 . In concert with this, there was a much smaller enhancement in the electron temperature at Cluster 2. Data from Cluster 1, 3 and 4 were of insufficient temporal resolution to determine whether or not there was an increase in the electron temperature at these spacecraft. Between 10:30 and 11:15 UT, Cluster detected 


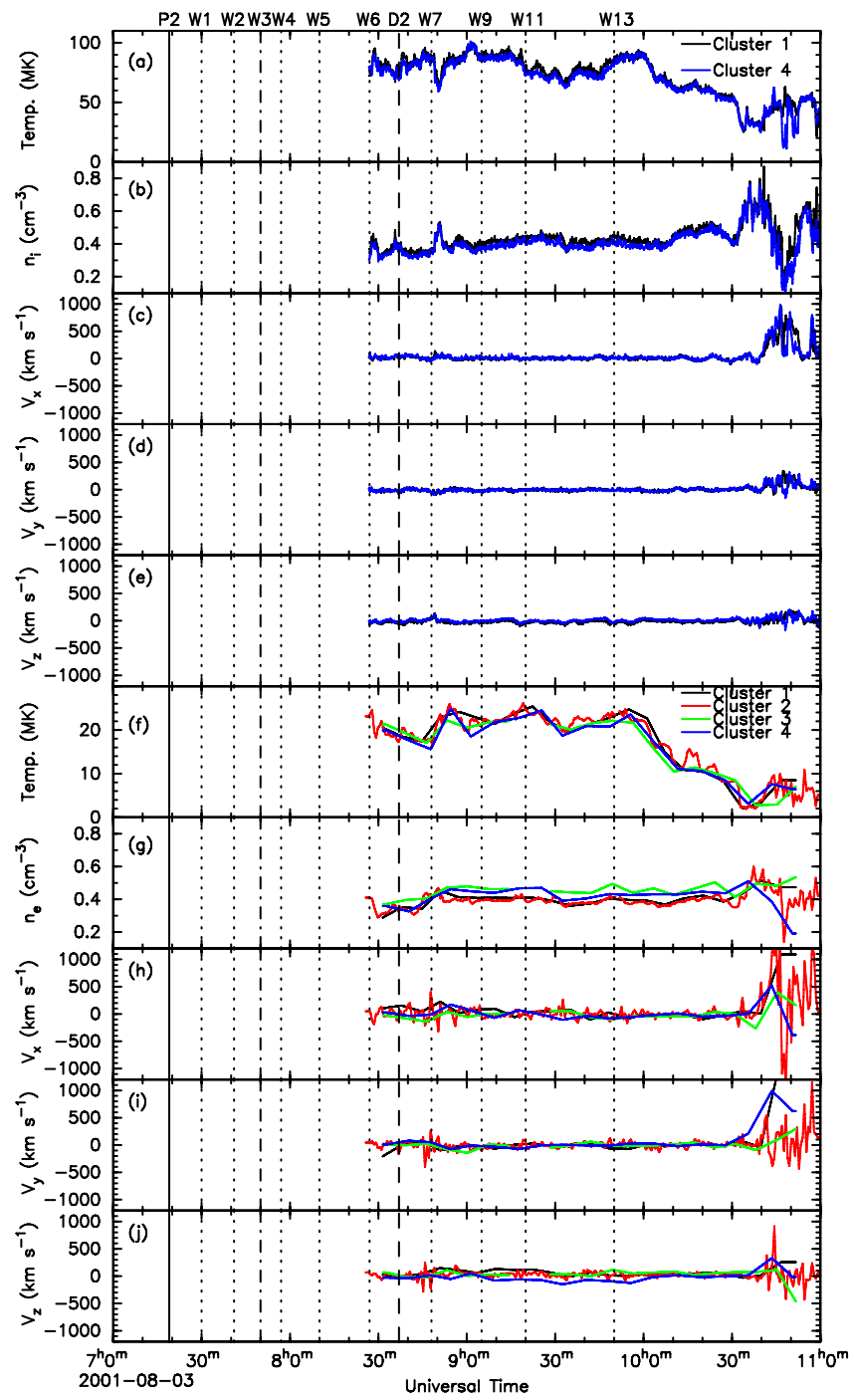

Fig. 9. Plot of particle moments from the Cluster CIS and PEACE instruments (Cluster 1: black, Cluster 2: red, Cluster 3: green, Cluster 4: blue). Panels (a-e) show ion moments from the CIS instruments. Panels $(\mathbf{f}-\mathbf{j})$ show moments from the PEACE instruments. The plots show (a-e and $\mathrm{f}-\mathrm{j}$, respectively) the temperature, density, $V_{X}, V_{Y}$ and $V_{Z}$ components.

a high-speed, lower density ion population, in concert with reversals in $B_{X}$ and $B_{Y}$ and an increased variability in all the magnetic field components. In particular, the high speed ions showed a large velocity perpendicular to the magnetic field in the Earthwards direction, indicating that Cluster detected a bursty bulk flow (BBF; Angelopoulos et al., 1992) and which was likely associated with the auroral substorm activity seen at this time (Fig. 6).

Figure 10 shows the average auroral luminosity between $55^{\circ}$ and $80^{\circ}$ magnetic latitude in bins of $1 \mathrm{~h}$ of MLT from the FUV-WIC onboard the IMAGE spacecraft. The MLT marked indicates the eastern edge of the bin, such that the

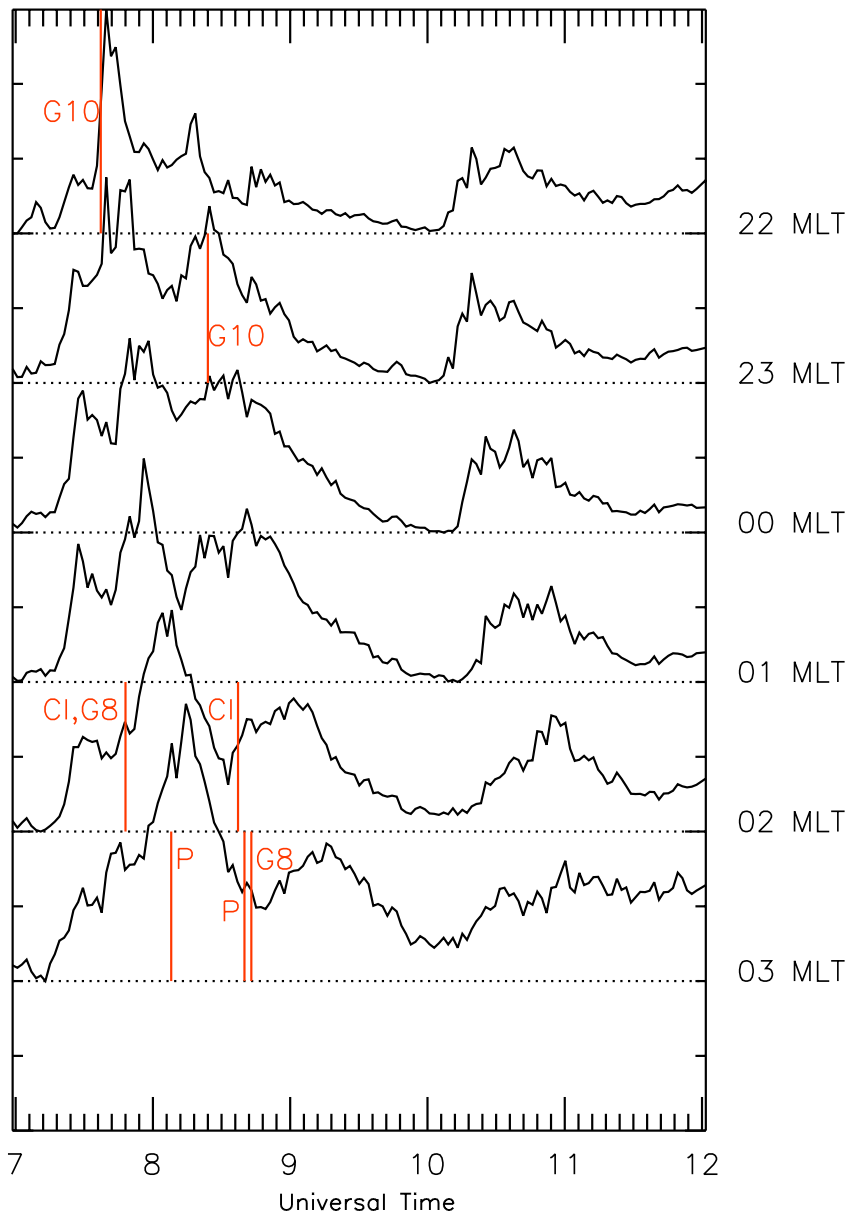

Fig. 10. Stack plot of auroral luminosity measured by FUV WIC on board the IMAGE spacecraft averaged over $1 \mathrm{~h}$ of MLT and between 55 to $80^{\circ}$ magnetic latitude. Each trace has had its minimum value subtracted, such that the dotted lines indicate the minimum value which has been set to $0 \mathrm{R}$. The traces are separated by $1500 \mathrm{R}$. The red lines indicate the times at which dipolarizations were detected by the Cluster $(\mathrm{Cl})$, GOES (G8 or G10) or Polar (P) spacecraft, indicated in the appropriate MLT sector.

01:00 MLT covers 01:00-02:00 MLT. The traces have been normalized by subtracting the minimum value from all the values for each trace. The traces are separated by $1500 \mathrm{R}$. The red lines indicate the times and MLT sectors in which the Cluster, GOES and Polar spacecraft detected dipolarizations of the magnetic field.

At 07:15 UT, the auroral luminosity across the night side began to increase, reaching a peak at 07:27 UT. The largest increase occurred between 23:00 and 02:00 MLT, where the average luminosity increased by $750-1000 \mathrm{R}$. Outside this range the increase was $\sim 500 \mathrm{R}$. At 07:40 UT there was an increase of over 1000 R in the 22:00 MLT sector accompanied by a $500 \mathrm{R}$ increase in the 23:00 MLT sector. This propagated eastward, with the peak of the brightening reaching the 
04:00 MLT sector by 08:18 UT before decaying to reach a local minimum at 08:48 UT. A further, smaller enhancement appeared at 08:18 UT in the 22:00 MLT sector and again propagated eastward, with the peak of the brightening reaching the 03:00 MLT sector by 09:12 UT before decaying to a minimum at 10:00 UT. A final enhancement, initiated in the 23:00 MLT sector at 10:12 UT, propagated both eastward and westward.

The red lines in Fig. 10 show that the dipolarizations detected by the Cluster and GOES spacecraft occurred just prior to the auroral enhancements, apart from the second dipolarization detected by GOES 10, indicating that the dipolarizations of the tail magnetic field were associated with the auroral substorms. The first dipolarization detected by Polar occurred at the time of peak brightness in the 03:00 MLT sector. Given this, and the much quicker dipolarization observed by Polar at this time, it seems unlikely that the dipolarization detected by Polar was the same feature detected by GOES.

Figure 10 shows that the enhancement of the aurora was less rapid as the aurora progressed towards dawn, hence determining the speed from the peaks of the auroral enhancements, which are well defined, would give a slower aurora expansion velocity than than if the velocity were determined from the start times of the enhancements. As such, we determine the velocity of the auroral expansion based on the start of the auroral enhancement in each sector. The eastward expansion velocities of the aurora were 5.5 and $2.75 \mathrm{kms}^{-1}$ for the first and second expansions respectively. Assuming that the dipolarization region expands in the $Y$ direction between GOES 10 and GOES 8 gives the dipolarization expansion velocities as 69 and $32 \mathrm{kms}^{-1}$ respectively. Mapping these velocities into the ionosphere from GOES 8 using the T89 model gives corresponding ionospheric velocities of 7 and $3 \mathrm{kms}^{-1}$, hence the auroral expansion velocities and the dipolarization region expansion velocities were similar.

Four-spacecraft timing analysis (Harvey, 1998) compares the lag of a given feature in the magnetic field across four points in space, and determines the velocity of the feature. For a planar discontinuity, the direction of travel is equivalent to the direction normal to the discontinuity. Assuming that the magnetic field variations are locally planar, in particular on the scale size of the Cluster tetrahedron, we apply these techniques to determine the propagation direction of the compression front and dipolarizations in the tail.

Table 1 shows the results of four-spacecraft timing analysis of the magnetic field data from Cluster for the effects of the pressure pulse ( $\mathrm{P} 2$ above) and for the dipolarization that is not directly associated with a current sheet wave (D2 above). The results from the four-spacecraft timing analysis are from a $10 \mathrm{~min}$ window centred on the time given. This window aids the comparison of the field between multiple spacecraft and visually checking the results. The data were smoothed using a $40 \mathrm{~s}$ Boxcar filter in order to remove any small scale fluctuations in the magnetic field. The lags between the spacecraft were determined from local minima and maxima in the $B_{X}$ component for the pressure pulse event and the $B_{Z}$ component for the dipolarizations. Results shown in italics were determined using EXT mode data from Cluster.

The results from the four-spacecraft timing analysis indicate that the normal to the compression front at Cluster (P2) was orientated predominantly in the $Z$ direction, whereas the dipolarization front was travelling in the $-Y$ direction and Earthward. Projecting the velocity of the dipolarization front into the ionosphere using the T89 model gives corresponding eastward ionospheric velocity of $2.5 \mathrm{kms}^{-1}$. This velocity is a good match for the observed auroral expansion velocity at this time and the projected ionospheric velocity from the GOES spacecraft.

In summary, data from the magnetometers onboard Cluster, Polar and GOES 8 and 10 have shown that prior to the solar wind pressure pulse the magnetotail was stretched due to the extended period of southward IMF. Following the arrival of the pressure pulse, the magnetosphere was compressed outside $6 R_{E}$ and the Region 1 currents were enhanced. The effects of the pressure pulse travelled downtail from GOES to Cluster, as we would expect for a travelling solar wind pressure front. Following the pressure pulse, dipolarizations were observed at GOES 10, GOES 8, and Polar at 07:35, 07:47, and 08:08 UT, respectively, and by GOES 8, GOES 10, Cluster and Polar at 08:25, 08:45, 08:37 and 08:40 UT, respectively. The dawnwards propagation of these dipolarizations between the GOES spacecraft and at Cluster was consistent with observations of dawnward moving aurora in the IMAGE FUV data, indicating that the dipolarizations were due to the occurrence of substorms and that at least one of the dipolarizations was observed across a distance of $12 R_{E}$ downtail.

\subsection{Current sheet wave observations}

Following the solar wind pressure pulse, Cluster observed multiple, pseudo-periodic reductions and recoveries in the $B_{X}$ component of the magnetic field, accompanied by local peaks in the field latitude angle starting at 07:30 UT (Fig. 8). After 08:48 UT, Cluster crossed the current sheet several times. These current sheet crossings have previously been studied by Zhang et al. (2002), who determined that they were due to current sheet waves. Given that $B_{X}$ reduces on approach to the current sheet, it is apparent the periodic reductions in $B_{X}$ prior to the current sheet crossings were also due to a wavy current sheet. Following the second substorm, Polar observed periodic decreases in the $B_{X}$ component of the magnetic field away from the elevated level with a similar form to those at Cluster. These are interpreted as the current sheet moving towards Polar, similar to the Cluster observations prior to the second substorm. These wave encounters at Cluster and Polar are labelled W1-13 in chronological order on Figs. 7 and 8.

Figure 9 shows that, following the second substorm, the current sheet wave had little effect on the plasma 
Table 1. The outputs of four-spacecraft timing analysis (4SCT) of the magnetic field data from Cluster. The clock angle (angle between the $\mathrm{Z}$ and $\mathrm{Y}$ components) of the 4SCT vector is given.

\begin{tabular}{cccccc}
\hline No. & Event & UT & $\begin{array}{c}\text { 4SCT } \\
(\mathrm{X}, \mathrm{Y}, \mathrm{Z})\end{array}$ & Speed & $\begin{array}{c}\text { Clock } \\
\left(\mathrm{kms}^{-1}\right)\end{array}$ \\
\hline P2 & Press. Pulse & $07: 19$ & $(0.06,0.33,-0.94)$ & 67 & 161 \\
D2 & Dipolarization & $08: 37$ & $(0.44,-0.76,-0.49)$ & 42 & -123 \\
\hline
\end{tabular}

environment at Cluster, with both ion and electron densities and velocities remaining approximately constant throughout the interval of the wave observations. The electron temperature increased by $5 \mathrm{MK}$ at the first wave encounter following the dipolarization D2 and remained at this elevated level until after the last current sheet crossing. Small variations in the ion $V_{Z}$ component were present, due to the bulk vertical motion of the current sheet and its plasma. These will be discussed further in Sect. 4.

Minimum variance analysis (MVA; Sonnerup and Cahill, 1967; Sonnerup and Scheible, 1998) can be used to determine the direction normal to a planar discontinuity or spatial variation in the magnetic field based on the assumption that magnetic field component normal to the discontinuity does not vary and that there is significant variation in the field in the plane of the discontinuity. In the ideal case, minimum variance analysis requires that at the magnetic field discontinuity, the two components of the magnetic field in the plane of the discontinuity vary to give a rotation of the field across the discontinuity and the magnetic field perpendicular to the discontinuity is invariant. In reality, small scale spatial and temporal variations mean that there is an apparent field variation perpendicular to the discontinuity, hence the ratio of the variances in the minimum and intermediate directions is used to indicate how well defined the minimum variance direction is. The MVA technique provides a variance matrix and associated eigenvalues, $\lambda_{1}, \lambda_{2}$ and $\lambda_{3}$, corresponding to the directions of maximum, intermediate and minimum variance respectively.

Sergeev et al. (2006) used data from the Cluster spacecraft to compare the results of MVA and four-spacecraft timing for 109 current sheet crossings. They found that when $\lambda_{2} / \lambda_{3}>4$ the vectors from the two techniques mostly agreed to within $30^{\circ}$. This indicates that, unlike the Harris current sheet model (Harris, 1962) which only has a direction of maximum variance, the tail current sheet has an intermediate variance direction which lies approximately in the direction of the cross-tail current and, as such, a minimum variance direction normal to the current sheet. Sergeev et al. related this variation in the intermediate direction to field-aligned currents or perturbations. Other effects, such as tail $B_{Y}$ components due to reconnection with the solar wind might also cause sufficient departure from the Harris model to introduce an intermediate variance direction. Since Polar is a single spacecraft, the MVA technique is used on data from Polar and Cluster to aid cross-comparison between them. Based on Sergeev et al. (2006), we will take $\lambda_{2} / \lambda_{3}>4$ to indicate that the MVA results are reliable.

Tables 2 and 3 list 13 current sheet oscillations from the Cluster and Polar datasets. Table 2 lists the results from MVA and four-spacecraft timing analysis from Cluster along with the angular difference between the vectors from the two techniques. Table 3 lists the MVA results from Polar along with the angular difference between the Polar MVA vectors and the Cluster MVA vectors and between the Polar MVA vectors and the Cluster four-spacecraft timing vectors.

The angles between the MVA and four-spacecraft timing vectors from the Cluster data indicate that the two techniques give similar results for the direction of the current sheet wave, with the vectors being within $\sim 10^{\circ}-20^{\circ}$ of each other. The angular difference between the two techniques and the $\lambda_{2} / \lambda_{3}$ ratios are in keeping with Sergeev et al. (2006). Comparing the MVA results from Polar with the MVA and fourspacecraft timing analysis results from Cluster shows that these vectors were not so well aligned, with the angle between the vectors varying between $45^{\circ}$ and $70^{\circ}$, despite the reasonably good $\lambda_{2} / \lambda_{3}$. However, upon closer inspection, it is obvious that all the vectors after 07:57 UT were directed dawnwards and Earthwards at both Cluster and Polar, apart from the MVA vector from W9 (Cluster) which has a tailward component. The largest variations between the Cluster and Polar vectors appears to be in the $Z$ component; the $Z$ components from the four-spacecraft timing analysis at Cluster all had a magnitude less than 0.33 and were predominantly negative, whereas the components from the MVA at Polar were generally larger than this but positive. Also, the $X$ component of the Polar MVA vectors was a factor of two or more larger.

Comparing the auroral activity (Fig. 10) to the observations of the current sheet waves, we note that the auroral luminosity in the 02:00 MLT sector reached a minimum shortly after the last current sheet crossing by Cluster. As such, the lifetime of the auroral luminosity and the wave activity at Cluster were similar.

To summarise; Cluster and Polar observed large, rapid changes in the magnetic field $B_{X}$ component following the 
Table 2. The outputs of the minimum variance analysis (MVA) and four spacecraft timing analysis (4SCT) of the magnetic field data from Cluster. The MVA vectors and eigenvalue ratios $\left(\lambda_{2} / \lambda_{3}\right)$ have been averaged across the four spacecraft. The clock angle (angle between the $\mathrm{Z}$ and $\mathrm{Y}$ components) of the 4SCT vector is given, along the the angular difference between the vectors from the two techniques.

\begin{tabular}{cccccccc}
\hline No. & UT & $\begin{array}{c}\text { MVA } \\
(\mathrm{X}, \mathrm{Y}, \mathrm{Z})\end{array}$ & $\lambda_{2} / \lambda_{3}$ & $\begin{array}{c}\text { 4SCT } \\
(\mathrm{X}, \mathrm{Y}, \mathrm{Z})\end{array}$ & $\begin{array}{c}\text { Speed } \\
\left(\mathrm{kms}^{-1}\right)\end{array}$ & Clock & Ang. diff \\
\hline W1 & $07: 30$ & $(0.23,0.11,0.97)$ & 5.61 & $(0.122,0.31,0.94)$ & 66 & 18 & 13 \\
W2 & $07: 41$ & $(0.19,0.31,0.93)$ & 4.74 & $(-0.14,0.09,0.99)$ & 82 & 5 & 23 \\
W3 & $07: 50$ & $(-0.27,-0.61,-0.74)$ & 2.03 & $(0.64,-0.77,0.04)$ & 80 & -87 & 74 \\
W4 & $07: 57$ & $(0.47,-0.86,0.17)$ & 1.81 & $(0.16,-0.98,0.08)$ & 70 & -85 & 21 \\
W5 & $08: 10$ & $(0.19,-0.97,0.13)$ & 19.7 & $(0.13,-0.94,0.32)$ & 61 & -71 & 12 \\
W6 & $08: 27$ & $(0.38,-0.92,0.11)$ & 12.0 & $(0.25,-0.93,-0.26)$ & 40 & -105 & 23 \\
W7 & $08: 48$ & $(0.41,-0.88,-0.24)$ & 4.47 & $(0.35,-0.89,-0.29)$ & 7 & -108 & 4 \\
W9 & $09: 05$ & $(-0.21,-0.98,0.05)$ & 3.4 & $(0.20,-0.94,-0.28)$ & 29 & -106 & 30 \\
W11 & $09: 20$ & $(0.22,-0.83,-0.51)$ & 12.8 & $(0.2,-0.94,-0.28)$ & 29 & -106 & 15 \\
W13 & $09: 50$ & $(0.30,-0.95,0.06)$ & 5.58 & $(0.64,-0.76,0.14)$ & 25 & -80 & 23 \\
\hline
\end{tabular}

Table 3. The outputs of the minimum variance analysis (MVA) of magnetic field data from the Polar spacecraft at various universal times along with the ratio of the intermediate to minimum eigenvalues. The Ang. Diff. MVA and Ang. Diff. 4SCT columns show the angular difference between the MVA vector at Polar and the MVA vector and four-spacecraft timing vector respectively from Cluster for the corresponding event (nearest in time).

\begin{tabular}{cccccc}
\hline No. & UT & $\begin{array}{c}\text { MVA } \\
(\mathrm{X}, \mathrm{Y}, \mathrm{Z})\end{array}$ & $\lambda_{2} / \lambda_{3}$ & $\begin{array}{c}\text { Ang. diff. } \\
\text { MVA }\end{array}$ & $\begin{array}{c}\text { Ang. diff. } \\
\text { 4SCT }\end{array}$ \\
\hline W8 & $08: 49$ & $(0.53,-0.57,0.631)$ & 4.55 & 56 & 59 \\
W10 & $09: 08$ & $(0.84,-0.54,-0.06)$ & 38.8 & 70 & 46 \\
W12 & $09: 25$ & $(0.38,-0.55,0.74)$ & 2.72 & 80 & 67 \\
\hline
\end{tabular}

solar wind pressure pulse, signalling the presence of current sheet waves. MVA and four-spacecraft timing analysis show that the current sheet waves were travelling consistently in the $-Y$ direction at Polar and Cluster. However, the $Z$ component of the normal to the current sheet had opposite senses at Cluster and Polar, such that the angle between the current sheet orientation at Cluster and Polar was large.

\section{Comparison of current sheet wave models}

Various models have been proposed to explain how the plasma sheet supports waves propagating perpendicular to the magnetic field. Early models based on MHD were unable to explain the relatively low speed of the cross-tail waves $\left(\sim 100 \mathrm{~km} \mathrm{~s}^{-1}\right)$ compared with the local sound speed $\left(\sim 1000 \mathrm{kms}^{-1}\right)$ whereas models based on ion drift fail to explain wave motion in the dawnward direction (Sergeev et al., 2004, and references therein). Golovchanskaya and Maltsev (2005) proposed a model based on the application of the ballooning instability waves (Safargaleev and Mal'Tsev, 1986) in a curved magnetic field. From the dispersion relation for ballooning perturbations,

$\omega^{2}=V_{A}^{2} k_{\|}^{2}+\omega_{g} \frac{k_{y}^{2}}{k_{n}^{2}+k_{y}^{2}}$

and using the assumptions made by Golovchanskaya and Maltsev, it can be shown that the phase velocity of the wave in the $Y$ direction is

$v_{y}^{p} \approx V_{A} \frac{\sqrt{2} B_{Z}+B_{X}^{\text {lobe }}}{B_{Z} a k_{y}}$

where $V_{A}$ is the Alfvén velocity of the current sheet particles, $B_{Z}$ is the $Z$ component of the magnetic field through the plasma sheet, $B_{X}^{\text {lobe }}$ is the $X$ component of the magnetic field in the lobe, $a$ is the current sheet half-thickness and $k$ is the wave vector in the $(\|)$ field-aligned, $(n)$ field-normal and $(Y)$ azimuthal directions.

Erkaev et al. (2008) considered that the Wentzel-KramersBrillouin (WKB) approximation used by Golovchanskaya and Maltsev was inappropriate for the plasma sheet. Instead, they proposed a model based on linearised MHD solved in a 

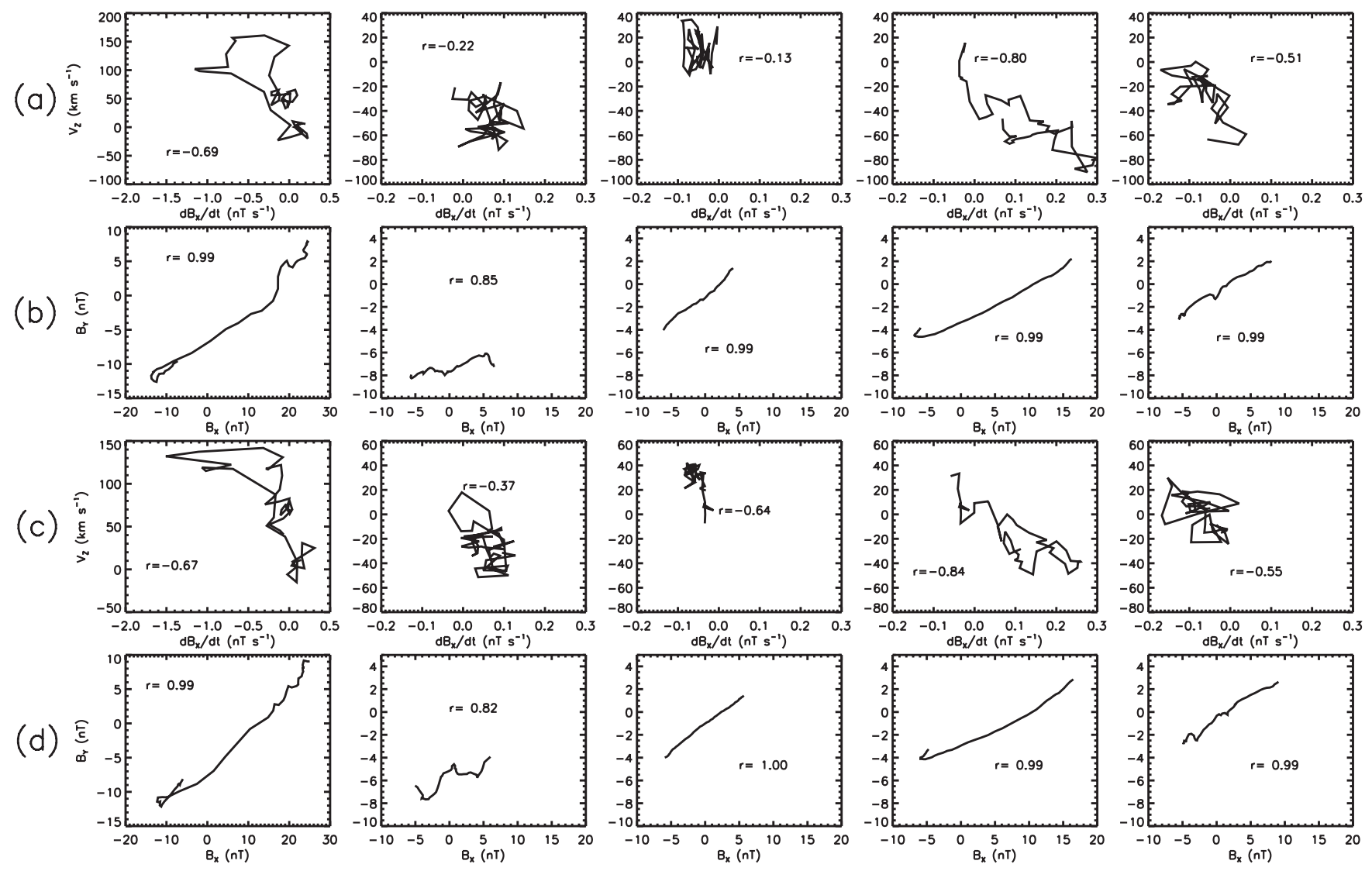

Fig. 11. Plots of $V_{Z}$ against $d B_{X} / d t$ (a and $\left.\mathbf{c}\right)$ and $B_{y}$ against $B_{X}$ (b and d) for each of the current sheet crossings detected by Cluster 1 (a and b) and Cluster 4 (c and d). The correlation coefficient is given in each panel.

piecewise manner from the centre to the edge of the current sheet then from the edge of the current sheet to the ionosphere. Across the current sheet, they allowed $B_{X}$ and $B_{Z}$ to vary with position. From this, they determined that the flapping frequency was given by

$\omega_{f}=\sqrt{\frac{1}{\mu_{0} \rho}\left\langle\frac{\partial B_{x}}{\partial z}\right) \frac{\partial B_{z}}{\partial x}}$

where $\rho$ is the current sheet density and the dispersion relation was

$\omega=\frac{\omega_{f} k \Delta}{\sqrt{k^{2} \Delta^{2}+\lambda_{k}^{2}}}$

where $\Delta$ is the current sheet half-thickness, $k$ is the wave number and $\lambda_{k}$ is the dimensionless numerical solution to $\tan \lambda_{k}=k \Delta / \lambda_{k}$. Note that the equations have been converted from the given form in Erkaev et al. (2008), in Gaussian units, to a form in SI units.

As both the above models can require the current sheet half-thickness as an input, we use the data from the Cluster spacecraft to determine the current sheet thickness for each of the current sheet crossings.
Sergeev et al. (1998) stated that, for a flapping current current sheet, $V_{Z}$ and $d B_{X} / d t$ would be correlated and that the current sheet half-thickness $(h)$ could be calculated as

$h=B_{l} A_{1} / \sqrt{1+k_{1}^{2}}$

where $B_{l}$ is the lobe magnetic field strength, $A_{1}$ is the gradient between $V_{Z}$ and $d B_{X} / d t$ and $k_{1}$ is the gradient between $B_{Y}$ and $B_{X}$. Figure 11 shows (a and c) $V_{Z}$ against $d B_{X} / d t$ and (b and d) $B_{Y}$ against $B_{X}$ for $200 \mathrm{~s}$ centred on the current sheet crossing for Cluster 1 and 4 respectively. The correlation coefficient is given in each panel. Table 4 shows the gradients between $V_{Z}$ and $d B_{X} / d t$ and $B_{Y}$ and $B_{Z}$ and the lobe magnetic field strengths determined by the Cluster spacecraft for each of the current sheet crossings. $B_{l}$ is calculated by assuming that the magnetic field pressure in the lobes balances the sum of the ion and magnetic pressures observed by Cluster.

Runov et al. (2005) showed that the distance of the barycentre of the Cluster spacecraft from the centre of the current sheet $\left(Z^{*}\right)$ could be calculated as

$Z *(t)=\int_{t_{1}}^{t_{2}} \frac{\partial B_{L}}{\partial t}\left[\nabla_{n} \cdot B_{L}\right]^{-1}$ 
(a)

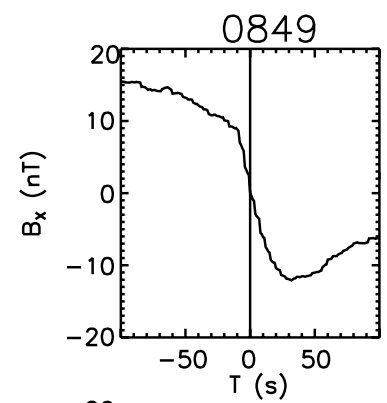

(b)

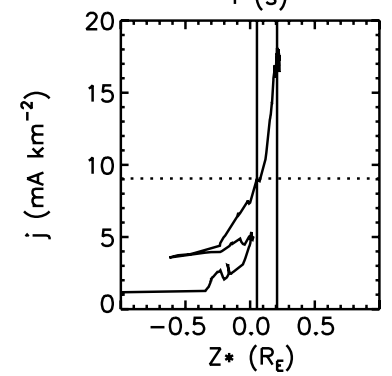

(c)

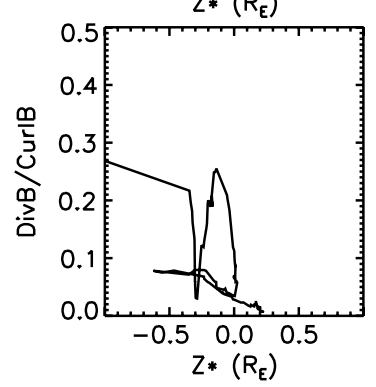

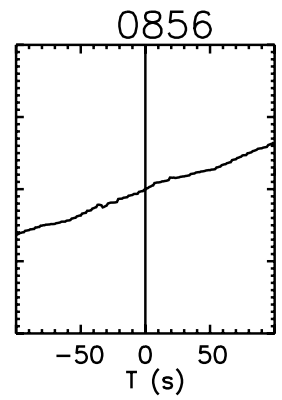
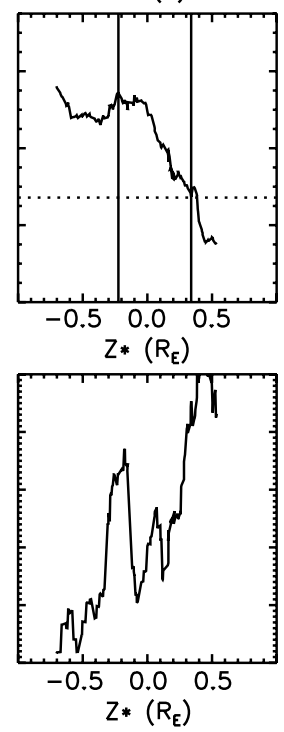
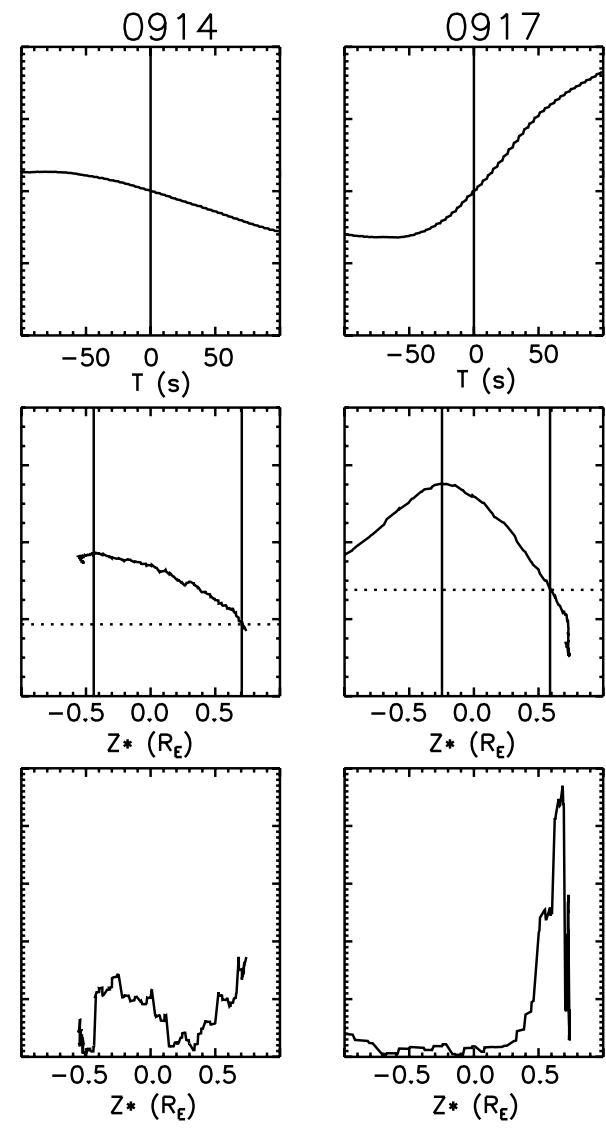
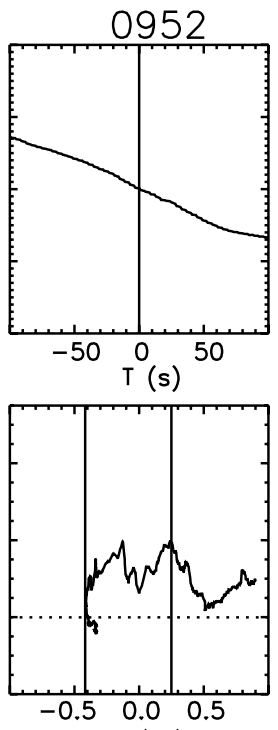

$Z *\left(R_{\varepsilon}\right)$

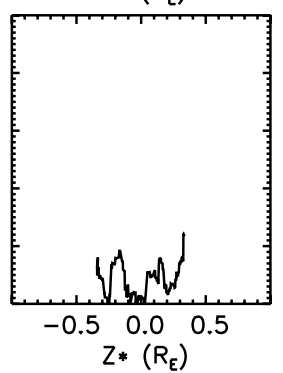

Fig. 12. Plots of (a) the average $B_{X}$ across the Cluster spacecraft, (b) the total current density against $Z^{*}$ and (c) DivB/CurlB against $Z^{*}$ for each of the current sheet crossings by Cluster. The times above the top row show the centre time $(t=0)$ of each column.

Table 4. The lobe magnetic field strength, the gradients between $V_{Z}$ and $d B_{X} / d t\left(A_{1}\right)$ and $B_{Y}$ and $B_{X}\left(k_{1}\right)$, and the current sheet halfthicknesses determined using the method of Sergeev et al. (1998) for the current sheet crossings by Cluster 1 (C1) and Cluster 4 (C4).

\begin{tabular}{ccccccccc}
\hline Crossing & $B_{l}(\mathrm{C} 1)$ & $A_{1}(\mathrm{C} 1)$ & $k_{1}(\mathrm{C} 1)$ & $\mathrm{CS}$ half-thickness $\left(R_{E}\right)$ & $B_{l}(\mathrm{C} 4)$ & $A_{1}(\mathrm{C} 4)$ & $k_{1}(\mathrm{C} 4)$ & $\mathrm{CS}$ half-thickness $\left(R_{E}\right)$ \\
\hline $08: 49$ & 33.29 & -99.53 & 0.50 & 0.46 & 32.70 & -82.12 & 0.54 & 0.37 \\
$08: 56$ & 30.00 & -79.72 & 0.13 & 0.37 & 28.66 & -139.2 & 0.28 & 0.60 \\
$09: 14$ & 29.79 & -71.37 & 0.49 & 0.30 & 28.77 & -418.4 & 0.46 & 1.71 \\
$09: 17$ & 30.26 & -202.3 & 0.28 & 0.92 & 29.21 & -185.3 & 0.28 & 0.82 \\
$09: 52$ & 27.13 & -179.5 & 0.37 & 0.72 & 25.96 & -160.2 & 0.40 & 0.61 \\
\hline
\end{tabular}

where $B_{L}$ is the barycentric magnetic field in the maximum variance direction (approximately the $X$ direction) and $\nabla_{n} . B_{L}$ is the gradient of the $B_{L}$ component in the direction perpendicular to the current sheet. The current sheet halfthickness can then be determined from the profile of the current density, $|\mathbf{j}|$, determined from the curlometer technique (Dunlop et al., 1988; Robert et al., 1998) against $Z^{*}$. Figure 12 shows (a) $B_{L}$, (b) the current density profile in the $Z^{*}$ direction and (c) the $|\operatorname{Div} B / \operatorname{Curl} B|$ profile in the $Z^{*}$ direc- tion. The dotted lines in (b) show half the maximum current density. The figure shows that the current sheet thickness varied with time and that for the majority of the current sheet crossings $|\operatorname{Div} B / \operatorname{Curl} B|$ was below 0.2 , indicating that the currents from the curlometer are reliable.

Figure 13 shows the current sheet half-thickness, determined from the above techniques, against time. The thicknesses from Cluster 1 and Cluster 4 from the Sergeev et al. technique are in black and red respectively, and the thickness 


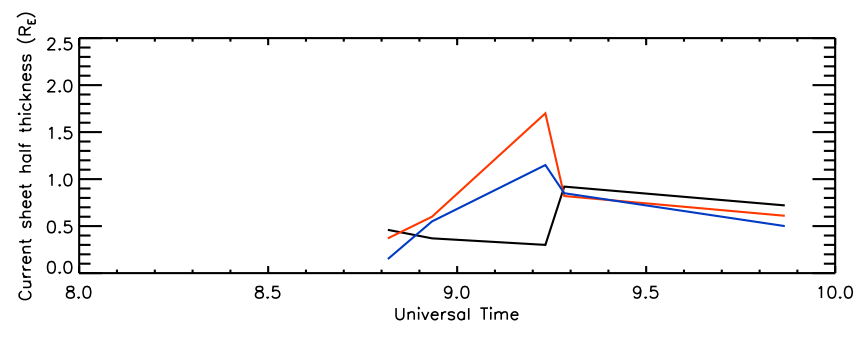

Fig. 13. Plot of current sheet half-thickness against time using the half-thicknesses determined by the Sergeev et al. technique (Cluster 1: black, Cluster 4: red) and the Runov et al. technique (blue).

from the Runov et al. technique is in blue. The two techniques give similar results for all the crossings, apart from the crossing at 09:14UT. For this crossing, the correlation coefficient between $V_{Z}$ and $d B_{Z} / d t$ from Cluster 1 was low, indicating that the gradient between them, and hence the current sheet thickness, was not well defined.

During the first current sheet crossing, the current sheet was very thin (half-thickness less than $0.5 R_{E}$ ). Subsequently, the current sheet thickened, reaching a maximum half-thickness of between 1 and $1.8 R_{E}$, before thinning again prior to the substorm at 10:15 UT.

Data from the PEACE and CIS-CODIF sensors (Fig. 9) show that the particle density during this interval was $\sim 0.4 \mathrm{~cm}^{-3}$. Using an ion population of $95 \% \mathrm{H}+$ and $5 \%$ $\mathrm{O}+$, as detected by the CIS-CODIF sensor, a lobe magnetic field in the $X$ direction of $40 \mathrm{nT}$, a sheet magnetic field of $25 \mathrm{nT}$ with a $B_{Z}$ of $15 \mathrm{nT}$, a current sheet Alfvén velocity of $650 \mathrm{kms}^{-1}$, a wave length of $4 R_{E}$ (from Zhang et al., 2002), and the average current sheet half-thicknesses from the above techniques (excluding the third value from Cluster 1 , which is clearly in error as discussed above), the phase velocity from the Golovchanskaya and Maltsev model were calculated to be between 1000 and $5000 \mathrm{kms}^{-1}$, which is two order of magnitudes larger than observed.

Using the mean magnetic field gradients determined by Cluster during $200 \mathrm{~s}$ surrounding each of the current sheet crossings and the same ion density as above, the wave periods in the Erkaev et al. model are determined to be between 2 to $8.5 \mathrm{~min}$. Using the average magnetic field gradients during the interval gives a wave period of $6 \mathrm{~min}$. These periods are somewhat smaller than the observed period of approximately $20 \mathrm{~min}$. Using the calculated wave frequencies, the wave speeds from the Erkaev et al. model were 44 to $130 \mathrm{kms}^{-1}$, although using a $20 \mathrm{~min}$ period gives velocities from 13 to $20 \mathrm{kms}^{-1}$, approximately two-thirds of the observed velocities.

Figure 14 shows the temporal variation of the velocity of the waves in the $Y_{\mathrm{GSM}}$ direction, determined from the fourspacecraft timing analysis of the Cluster data, along with the phase velocities calculated from the Erkaev et al. model for a

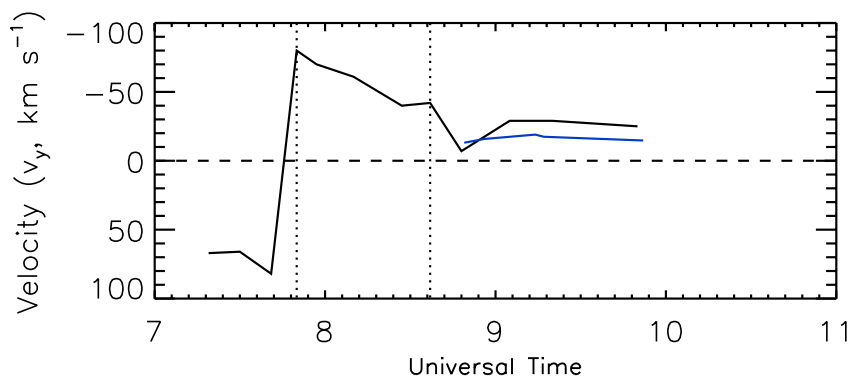

Fig. 14. Plot of the velocity in the $Y$ direction (black), determined from four-spacecraft analysis, for the dipolarization and wave fronts against time and the phase velocity calculated from the Erkaev et al. (2008) model using the observed current sheet half-thicknesses and a wave period of $20 \mathrm{~min}$. The dashed lines indicate the times of the dipolarizations.

wave period of $20 \mathrm{~min}$ (blue trace). The observed and model velocities follow similar trends, although the model velocities are approximately two-thirds of the observed velocities, as noted above. It should be noted that the true phase velocity of these waves should be across the current sheet, whereas the observed velocity is the velocity perpendicular to the wave front. Given that the current sheet was in motion at this time, it is difficult to determine its average inclination in the $Y Z$ plane. As such, the velocity of the waves in the $Y$ direction is taken as an approximation to the phase velocity of the waves.

\section{Discussion}

\subsection{Magnetospheric compression and magnetotail dipo- larizations}

In this study, a solar wind pressure pulse, following an extended period of southward IMF, was observed to compress the magnetosphere prior to the onset of three substorms. Subsequent to the arrival of the solar wind pressure pulse at the dayside, the magnetic field magnitude at Cluster's location in the tail increased by a factor of $\sim 2$, dominated by an increase in the $B_{X}$ component (Fig. $8 \mathrm{a}$ and d). Data from GOES 8 and 10 also showed increases in the magnetic field magnitude at this time (Fig. 7) associated with an enhancement in the Region 1 currents. Polar observed a circularly polarised wave inside of $5 R_{E} X_{\mathrm{GSM}}$ at this time, with a period of $\sim 2.5 \mathrm{~min}$. The solar wind pressure pulse excited global auroral activity. Data from FUV-WIC on board the IMAGE spacecraft showed that, following the solar wind pressure pulse, the average luminosity in the nightside MLT sectors increased by up to $1000 \mathrm{R}$, with the largest increase occurring from 23:00 to 03:00 MLT.

Following the pressure pulse, three substorm expansion phases, starting at 07:35, 08:18 and 10:15 UT respectively, 
were observed in auroral and ground magnetometer data (Fig. 6). In the magnetosphere, GOES 8 and 10, Polar and Cluster all observed dipolarizations of the magnetic field. At Cluster, the first dipolarization was observed in tandem with a current sheet wave. At Polar, the first dipolarization was much more rapid that at the other spacecraft. During the final substorm, Cluster detected a bursty bulk flow (Angelopoulos et al., 1992) and crossed the magnetotail current sheet twice.

The eastward expansion velocities of the first two auroral substorms were well matched by the projected ionospheric velocities of the dipolarizations from GOES. Mapping the propagation of the dipolarization across the Cluster spacecraft into the ionosphere also gave an ionospheric velocity of the footpoint of the dipolarization comparable to the auroral expansion velocity. This indicates that the region of dipolarized magnetic field associated with the second substorm extended over $12 R_{E}$ and expanded eastwards. These observations are consistent with Liou et al. (2002) and Nakamura et al. (2005) who showed the correlation between the motion of an auroral substorm and dipolarizations at geostationary orbit and the detection of a dipolarization front across $5 R_{E}$ respectively. The eastward motion of the aurora is also consistent with the IMF $B_{Y}$ control of the motion of the auroral bulge (Liou et al., 2006; Liou and Ruohoniemi, 2006a,b).

\subsection{Current sheet wave}

Following the solar wind pressure pulse, Cluster and Polar observed large, rapid decreases and recoveries in the magnetic field magnitude, dominated by variations in the $B_{X}$ component and accompanied by increases in the latitude angle. The variations in the field at Polar were not observed until after the second substorm. In a previous study of this time, Zhang et al. (2002) showed that, after 08:48 UT, the variations in the magnetic field at Cluster were due to Cluster crossing the current sheet several times. They related these current sheet crossings to a current sheet wave and used four-spacecraft timing analysis on the $B_{X}=0 \mathrm{nT}$ crossings to determine that the wave was travelling in the $-Y$ direction. We have expanded upon this study, incorporating data from a larger dataset and including data from the Polar spacecraft. Although prior to 08:48 UT Cluster did not cross the current sheet, the periodic reductions in the $B_{X}$ component indicate that the current sheet wave was present at this time. During the third substorm, Cluster crossed the current sheet twice during the passage of a bursty bulk flow. It is clear from the particle moments and the magnetic field data that the plasma dynamics during these crossings were very different from the current sheet wave activity beforehand. As such, these crossings are not considered within this study.

Only three out of the nine Cluster observations of the current sheet wave had values of $\lambda_{2} / \lambda_{3}<4$. In keeping with Sergeev et al. (2006), the angles between the vectors from the MVA and four-spacecraft timing analysis were fairly low. However, comparing the angle between the MVA vectors at
Polar and the MVA and four-spacecraft timing analysis vectors at Cluster shows that the vectors are consistently out by $>40^{\circ}$ despite one of the events having a particularly high $\lambda_{2} / \lambda_{3}$. This would suggest that there was some offset between the Polar and Cluster vectors, most likely due to their large separation. Comparing the vectors directly shows that the $Y$ components are fairly consistent but the angle in the $\mathrm{XZ}$ plane is oppositely directed at one spacecraft compared with the other. The results of the MVA and four-spacecraft timing analysis at Cluster (Table 2) and MVA at Polar (Table 3) has shown that for all the waves observed following the substorms, the waves were travelling in the $-Y$ direction, although it is not possible to determine whether or not the waves at Polar were travelling in this direction or the opposite direction unambiguously. However, given that the dipolarization fronts and auroral enhancements were travelling dawnwards, it seems reasonable to suggest that the same is true for the current sheet wave in the vicinity of Polar.

Despite FGM EXT mode being designed to provide contextual information and not usually suited to multi-spacecraft analysis (Brown et al., 2008), comparison between the MVA and four-spacecraft timing analysis of the data and comparison of the results of these techniques between the EXT mode data and $5 \mathrm{~Hz}$ CAA data would suggest that the results, in this case, are reliable, given the similarity between the vectors obtained at different times and the relatively small angle between the vectors from the two different techniques.

Prior to the first substorm, Cluster observed periodic variations in the magnetic field with phase fronts orientated predominantly in the $Z$ direction. The orientation of these variations was more in keeping with the orientation of the compression front observed at 07:19UT. It is unclear whether this oscillation of the magnetosphere prior to the first substorm is the generation mechanism for the current sheet wave and that the substorms acted to modify the wave's propagation, or whether the substorms introduced a new wave into the system.

That the spacecraft did not cross the current sheet following the first substorm may be due to several factors. Using the change in $B_{X}$ as a proxy for the amplitude of the oscillation, it appears that the amplitude increased following each substorm. As such, the amplitude of the oscillation may have only become sufficient following the second substorm to cause the spacecraft to cross the current sheet. Alternatively, as Cluster was moving towards the current sheet from the northern part of the magnetotail, Cluster would have approached closer to the average position of the centre of the current sheet between the two substorms, such that Cluster was close enough to cross the current sheet following the second substorm. Petrukovich et al. (2006) noted that the wave amplitude increased with increasing tilt of the current sheet for a fortuitous event on 3 August 2004. This does not appear to be the case for the oscillations studied here. 


\subsection{Comparison of current sheet wave models}

The observations of the current sheet waves were used to compare the current sheet wave models of Golovchanskaya and Maltsev (2005) and Erkaev et al. (2008). As both models use the current sheet thickness in their calculation of further parameters, it was necessary to determine the current sheet thickness. This was determined in two ways; by correlating $V_{Z}$ with $d B_{X} / d t$ and $B_{Y}$ and $B_{X}$ (Sergeev et al., 1998 ) and by determining the temporal and spatial variations of the field Runov et al. (2005). Although the correlation coefficient between $V_{Z}$ and $d B_{X} / d t$ was less than 0.3 for two of the current sheet crossings, and DivB/CurlB was significantly higher for the same event (indicating a poorer determination of $\mathbf{j}$ and $\nabla \mathbf{B}$ ), Fig. 13 shows that the two methods gave similar results for the current sheet thicknesses during each of the crossings. It also appears that the current sheet was thickening between 08:49 and 09:17 UT before thinning slightly.

Using the model of Golovchanskaya and Maltsev (2005), the calculated velocities were found to be two orders of magnitude larger than the observed velocities. In contrast, the wave velocities from the model of Erkaev et al. (2008) were close to those observed and that followed the same trend (Fig. 14). As such, the Erkaev et al. model appears to be more applicable in this situation. It is worth noting, however, that using the Erkaev et al. model to calculate the expected wave frequencies using the observed magnetic field gradients returned values that an order of magnitude smaller than observed. This may represent an over simplification of the tail magnetic field topology used by Erkaev et al. (2008) or may indicate that the observed magnetic field gradients are not indicative of the global properties of the magnetosphere. The most likely explanation is that it is some combination of both effects.

Both Golovchanskaya and Maltsev (2005) and Erkaev et al. (2008) used their models to determine the group velocity of the waves and compared this to the observed velocities reported in previous studies (e.g. Sergeev et al., 2004). Although the group velocity may give us more information about the energy transported in the waves, by the nature of the observations, the observed velocity must be the phase velocity of the waves. It is clear from the dispersion relations that the phase and group velocities are not equal in these models. As such, we compared the phase velocities from these models with the observations.

Neither of the models discussed consider the generation mechanism for the flapping motion of the plasma sheet, only the mechanism that supports the wave propagation. Erkaev et al. (2008) suggested that BBFs created by reconnection events may excite the wave activity "like a ship moving on a water surface". This is consistent with a near-Earth neutral line injecting Earthward-moving flux into the plasma sheet (Cowley, 1984), such as is expected during substorms, and exciting the wave.
Our results suggest that there is a strong link between the substorm expansion phase and the wavy current sheet, clearly showing that the current sheet wave and expansion of the region of dipolarized magnetic field had comparable velocities, that the cross tail current sheet wave occurred after the substorm onsets and that the lifetime of the auroral enhancements was comparable with the lifetime of the current sheet waves. However, our results also show the presence of a periodic variation orientated in the $Z$ direction in the magnetotail prior to the substorm onset. It is not clear what effect this earlier wave had on the generation of the current sheet wave. One possibility is that the excitation of the wavy current sheet occurs in two parts. Firstly, the solar wind pressure pulse compresses the magnetosphere, and hence the current sheet. This causes the current sheet to oscillate in the $Z_{\mathrm{GSM}}$ direction. Following this, two substorms dipolarize the mid-tail. This disruption in the mid-tail changes the magnetic field oscillation from an oscillation in the $Z_{\mathrm{GSM}}$ direction to a wave propagating in the $-Y_{\mathrm{GSM}}$ direction with approximately the same period, with the amplitude of the oscillation increasing following each substorm. However, the model of Erkaev et al. shows that the wave period is dependent on the tail configuration and it is unclear what effect, if any, a pre-existing wave would have.

The similarity between the propagation velocities of the current sheet waves, the two dipolarization expansions and the expanding aurora may indicate that the propagation velocities of these features are controlled by the configuration of the tail defined as in the Erkaev et al. model. Further observations of these features that consider these constraints are required to test this hypothesis.

\section{Conclusions}

Two substorms following a solar wind pressure pulse on 3 August 2001, have been examined in the context of the wavy current sheet, as investigated by Zhang et al. (2002). In both cases, the dipolarization was seen at geosynchronous orbit by GOES 8 and 10 and Polar, and by the Cluster spacecraft in the tail. We have shown that the projected ionospheric velocities of the expansion of the region of dipolarized magnetic field were consistent with the motion of the substorm auroral activity, confirming that the expansion of the region of dipolarized magnetic field is related to substorm auroral activity and indicating that this is true across large distances in the tail.

Following both substorm onsets, Cluster and Polar detected signatures of a wavy current sheet. Using MVA and four-spacecraft timing analysis, it has been shown that the dipolarizations and current sheet waves propagated across the tail with similar velocities. Our results are not able to determine whether or not the substorms were the source of the wave, since there is evidence of an oscillation of the plasma 
sheet orientated in the Z-direction following the solar wind pressure pulse.

We have tested the models of Golovchanskaya and Maltsev (2005) and Erkaev et al. (2008) against our observations. We found that the model of Golovchanskaya and Maltsev (2005) over-estimated the phase velocity of the waves by two orders of magnitude compared with the observations. In contrast, the model of Erkaev et al. (2008) reproduced the expected wave velocity to within two-thirds of the observed wave velocities. Based on this, and the similar velocities of the dipolarization fronts and the current sheet waves, we have suggested that the propagation velocities of dipolarization fronts of substorm expansion phase and the current sheet wave are determined by the magnetotail configuration.

Acknowledgements. The authors wish to thank CDAWeb for the GOES data; H. U. Frey and the NASA Space Science Data Centre for the IMAGE FUV data; the Cluster Active Archive for the high resolution FGM data and CIS data; C. T. Russell for the Polar MFE data; N. Ness and D. McComas for the ACE data. We thank the institutes who maintain the IMAGE Magnetometer array. Thanks go to the operations teams of the various instruments and spacecraft used in this study. Analysis of the Cluster, GOES and Polar data was done using the QSAS science analysis system provided by the UK Cluster Science Centre (Imperial College London and Queen Mary, University of London). ML, RCF and TKY were supported by STFC grant PP/E000983. CF was supported by STFC studentship PPA/S/S/2005/04156 and STFC grant PP/E/001173/1.

Topical Editor I. A. Daglis thanks two anonymous referees for their help in evaluating this paper.

\section{References}

Angelopoulos, V., Baumjohann, W., Kennel, C. F., Coronti, F. V., Kivelson, M. G., Pellat, R., Walker, R. J., Luehr, H., and Paschmann, G.: Bursty bulk flows in the inner central plasma sheet, J. Geophys. Res., 97, 4027-4039, 1992.

Baker, K. B. and Wing, S.: A new magnetic coordinate system for conjugate studies at high latitudes, J. Geophys. Res., 94, 91399143, 1989.

Balogh, A., Carr, C. M., Acuña, M. H., Dunlop, M. W., Beek, T. J., Brown, P., Fornacon, K.-H., Georgescu, E., Glassmeier, K.H., Harris, J., Musmann, G., Oddy, T., and Schwingenschuh, K.: The Cluster Magnetic Field Investigation: overview of in-flight performance and initial results, Ann. Geophys., 19, 1207-1217, 2001, http://www.ann-geophys.net/19/1207/2001/.

Bauer, T. M., Baumjohann, W., and Treumann, R. A.: Neutral sheet oscillations at substorm onset, J. Geophys. Res., 100, 2373723742, doi:10.1029/95JA02448, 1995.

Brown, P., Dunlop, M. W., Balogh, A., Carr, C., Gloag, J., Lucek, E., and Oddy, T.: Calibration techniques for magnetometers implementing on-board de-spinning algorithms, Adv. Space Res., 41, 1571-1578, doi:10.1016/j.asr.2007.09.028, 2008.

Cowley, S. W. H.: The Distant Geomagnetic Tail in Theory and Observation, in: Magnetic Reconnection in Space and Laboratory Plasmas, edited by: Hones, Jr., E. W., pp. 228-239, 1984.

Dunlop, M. W., Southwood, D. J., Glassmeier, K.-H., and Neubauer, F. M.: Analysis of multipoint magnetometer data,
Adv. Space Res., 8, 273-277, doi:10.1016/0273-1177(88) 90141-X, 1988.

Erkaev, N. V., Semenov, V. S., and Biernat, H. K.: Magnetic double gradient mechanism for flapping oscillations of a current sheet, Geophys. Res. Lett., 35, L02111, doi:10.1029/2007GL032277, 2008.

Fairfield, D. H. and Ness, N. F.: Configuration of the geomagnetic tail during substorms., J. Geophys. Res., 75, 7032-7047, 1970.

Golovchanskaya, I. V. and Maltsev, Y. P.: On the identification of plasma sheet flapping waves observed by Cluster, Geophys. Res. Lett., 32, L02102, doi:10.1029/2004GL021552, 2005.

Harris, E. G.: On a plasma sheath separating regions of oppositely directed magnetic field, Il Nuovo Cimento, 23, 115-121, doi: 10.1007/BF02733547, 1962.

Harvey, C. C.: Spatial gradients and the volumetric tensor, in: Analysis Methods for Multi-Spacecraft Data, edited by: Paschmann, G. and Daly, P. W., pp. 307-348, ISSI, 1998.

Johnstone, A. D., Alsop, C., Burge, S., Carter, P. J., Coates, A. J., Coker, A. J., Fazakerley, A. N., Grande, M., Gowen, R. A., Gurgiolo, C., Hancock, B. K., Narheim, B., Preece, A., Sheather, P. H., Winningham, J. D., and Woodliffe, R. D.: PEACE: a Plasma Electron And Current Experiment, Space Sci Rev., 79, 351-398, 1997.

Khan, H. and Cowley, S. W. H.: Observations of the response time of high-latitude ionospheric convection to variations in the interplanetary magnetic field using EISCAT and IMP-8 data, Ann. Geophys., 17, 1306-1335, 1999, http://www.ann-geophys.net/17/1306/1999/.

Kokubun, S. and McPherron, R. L.: Substorm signatures at synchronous altitude, J. Geophys. Res, 86, 11265-11277, 1981.

Liou, K. and Ruohoniemi, J. M.: A case study of relationship between substorm expansion and global plasma convection, Geophys. Res. Lett., 33, L02105, doi:10.1029/2005GL024736, 2006a.

Liou, K. and Ruohoniemi, J. M.: Correction to "A case study of relationship between substorm expansion and global plasma convection", Geophys. Res. Lett., 33, L10101, doi:10.1029/ 2006GL025990, 2006b.

Liou, K., Meng, C.-I., Lui, A. T. Y., Newell, P. T., and Wing, S.: Magnetic dipolarization with substorm expansion onset, J. Geophys. Res., 107, 1131-1142, doi:10.1029/2001JA000179, 2002.

Liou, K., Meng, C.-I., and Wu, C.-C.: On the interplanetary magnetic field $\mathrm{B}_{y}$ control of substorm bulge expansion, J. Geophys. Res., 111, 9312-9319, doi:10.1029/2005JA011556, 2006.

Luhr, H.: The IMAGE Magnetometer Network, in: STEP International, vol. 4, pp. 4-6, USSCO, 1994.

McComas, D. J., Bame, S. J., Barker, P., Feldman, W. C., Phillips, J. L., Riley, P., and Griffee, J. W.: Solar Wind Electron Proton Alpha Monitor (SWEPAM) for the Advanced Composition Explorer, Space Sci. Rev., 86, 563-612, doi:10.1023/A: 1005040232597, 1998.

McPherron, R. L., Russell, C. T., and Aubry, M. P.: Satellite studies of magnetospheric substorms on August 15, 1968. 9. Phenomenological model for substorms., J. Geophys. Res., 78, 3131-3149, 1973.

Mende, S. B., Heetderks, H., Frey, H. U., Lampton, M., Geller, S. P., Abiad, R., Siegmund, O. H. W., Tremsin, A. S., Spann, J., Dougani, H., Fuselier, S. A., Magoncelli, A. L., Bumala, M. B., Murphree, S., and Trondsen, T.: Far ultraviolet imaging from the 
IMAGE spacecraft. 2. Wideband FUV imaging, Space Sci. Rev., 91, 271-285, 2000a.

Mende, S. B., Heetderks, H., Frey, H. U., Lampton, M., Geller, S. P., Habraken, S., Renotte, E., Jamar, C., Rochus, P., Spann, J., Fuselier, S. A., Gerard, J.-C., Gladstone, R., Murphree, S., and Cogger, L.: Far ultraviolet imaging from the IMAGE spacecraft. 1. System design, Space Sci. Rev., 91, 243-270, 2000 b.

Mende, S. B., Heetderks, H., Frey, H. U., Stock, J. M., Lampton, M., Geller, S. P., Abiad, R., Siegmund, O. H. W., Habraken, S., Renotte, E., Jamar, C., Rochus, P., Gerard, J.-C., Sigler, R., and Lauche, H.: Far ultraviolet imaging from the IMAGE spacecraft. 3. Spectral imaging of Lyman- $\alpha$ and OI $135.6 \mathrm{~nm}$, Space Sci. Rev., 91, 287-318, 2000c.

Nagai, T.: Observed magnetic substorm signatures at synchronous altitude, J. Geophys. Res., 87, 4405-4417, 1982.

Nakagawa, T. and Nishida, A.: Southward magnetic field in the neutral sheet produced by wavy motions propagating in the dawndusk direction, Geophys. Res. Lett., 16, 1265-1268, 1989.

Nakamura, R., Baumjohann, W., Zhang, T. L., Carr, C. M., Balogh, A., Fornacon, K-H., Georgescu, E., Rme, H., Dandouras, I., Takada, T., Volwerk, M., Asano, Y., Runov, A., Eichelberger, H., Klecker, B., Mouikis, C., Kistler, L. M., and Amm, O.: Cluster and Double Star observations of dipolarization, Ann. Geophys., 23, 2915-2920, 2005,

http://www.ann-geophys.net/23/2915/2005/.

Petrukovich, A. A., Zhang, T. 1., Baumjohann, W., Nakamura, R., Runov, A., Balogh, A., and Carr, C.: Oscillatory magnetic flux tube slippage in the plasma sheet, Ann. Geophys., 24, 16951704, 2006, http://www.ann-geophys.net/24/1695/2006/.

Rème, H., Aoustin, C., Bosqued, J. M., Dandouras, I., Lavraud, B., Sauvaud, J. A., Barthe, A., Bouyssou, J., Camus, Th., CoeurJoly, O., Cros, A., Cuvilo, J., Ducay, F., Garbarowitz, Y., Medale, J. L., Penou, E., Perrier, H., Romefort, D., Rouzaud, J., Vallat, C., Alcaydé, D., Jacquey, C., Mazelle, C., dUston, C., Möbius, E., Kistler, L. M., Crocker, K., Granoff, M., Mouikis, C., Popecki, M., Vosbury, M., Klecker, B., Hovestadt, D., Kucharek, H., Kuenneth, E., Paschmann, G., Scholer, M., Sckopke, N., Seidenschwang, E., Carlson, C. W., Curtis, D. W., Ingraham, C., Lin, R. P., McFadden, J. P., Parks, G. K., Phan, T., Formisano, V., Amata, E., Bavassano-Cattaneo, M. B., Baldetti, P., Bruno, R., Chionchio, G., Di Lellis, A., Marcucci, M. F., Pallocchia, G., Korth, A., Daly, P. W., Graeve, B., Rosenbauer, H., Vasyliunas, V., McCarthy, M., Wilber, M., Eliasson, L., Lundin, R., Olsen, S., Shelley, E. G., Fuselier, S., Ghielmetti, A. G., Lennartsson, W., Escoubet, C. P., Balsiger, H., Friedel, R., Cao, J.-B., Kovrazhkin, R. A., Papamastorakis, I., Pellat, R., Scudder, J., and Sonnerup, B.: First multispacecraft ion measurements in and near the Earth's magnetosphere with the identical Cluster ion spectrometry (CIS) experiment, Ann. Geophys., 19, 1303-1354, 2001, http://www.ann-geophys.net/19/1303/2001/.

Robert, P., Dunlop, M. W., Roux, A., and Chanteur, G.: Accuracy of Current Density Determination, in: Analysis Methods for MultiSpacecraft Data, edited by: Paschmann, G. and Daly, P. W., pp. 395-418, ISSI, 1998.

Rostoker, G.: Current flow in the magnetosphere during magnetospheric substorms., J. Geophys. Res., 79, 1994-1998, 1974.

Runov, A., Nakamura, R., Baumjohann, W., Zhang, T. L., Volwerk, M., Eichelberger, H.-U., and Balogh, A.: Cluster observation of a bifurcated current sheet, Geophys. Res. Lett., 30(2), L01036,
doi:10.1029/2002GL016136, 2003.

Runov, A., Sergeev, V. A., Baumjohann, W., Nakamura, R., Apatenkov, S., Asano, Y., Volwerk, M., Vrs, Z., Zhang, T. L., Petrukovich, A., Balogh, A., Sauvaud, J.-A., Klecker, B., and Rme, H.: Electric current and magnetic field geometry in flapping magnetotail current sheets, Ann. Geophys., 23, 1391-1403, 2005, http://www.ann-geophys.net/23/1391/2005/.

Runov, A., Sergeev, V. A., Nakamura, R., Baumjohann, W., Apatenkov, S., Asano, Y., Takada, T., Volwerk, M., Vrs, Z., Zhang, T. L., Sauvaud, J.-A., Rème, H., and Balogh, A.: Local structure of the magnetotail current sheet: 2001 Cluster observations, Ann. Geophys., 24, 247-262, 2006, http://www.ann-geophys.net/24/247/2006/.

Russell, C. T., Snare, R. C., Means, J. D., Pierce, D., Dearborn, D., Larson, M., Barr, G., and Le, G.: The GGS/POLAR magnetic fields investigation, Space Sci. Rev., 71, 563-582, doi:10.1007/ BF00751341, 1995.

Safargaleev, V. V. and Mal'Tsev, Y. P.: Internal gravitational waves in a plasma sheet., Geomagnetism and Aeronomy, 26, 270-274, 1986.

Sergeev, V., Angelopoulos, V., Carlson, C., and Sutcliffe, P.: Current sheet measurements within a flapping plasma sheet, J. Geophys. Res., 103, 9177-9188, doi:10.1029/97JA02093, 1998.

Sergeev, V., Runov, A., Baumjohann, W., Nakamura, R., Zhang, T. L., Volwerk, M., Balogh, A., Rème, H., Sauvaud, J. A., André, M., and Klecker, B.: Current sheet flapping motion and structure observed by Cluster, Geophys. Res. Lett., 30(6), L01327, doi:10.1029/2002GL016500, 2003.

Sergeev, V., Runov, A., Baumjohann, W., Nakamura, R., Zhang, T. L., Balogh, A., Louarnd, P., Sauvaud, J.-A., and Reme, H.: Orientation and propagation of current sheet oscillations, Geophys. Res. Lett., 31, L05807, doi:10.1029/2003GL019346, 2004

Sergeev, V. A., Sormakov, D. A., Apatenkov, S. V., Baumjohann, W., Nakamura, R., Runov, A. V., Mukai, T., and Nagai, T.: Survey of large-amplitude flapping motions in the midtail current sheet, Ann. Geophys., 24, 2015-2024, 2006, http://www.ann-geophys.net/24/2015/2006/.

Smith, C. W., L'Heureux, J., Ness, N. F., Acuña, M. H., Burlaga, L. F., and Scheifele, J.: The ACE Magnetic Fields Experiment, Space Sci. Rev., 86, 613-632, doi:10.1023/A:1005092216668, 1998.

Sonnerup, B. U. O. and Cahill Jr., L. J.: Magnetopause structure and attitude from Explorer 12 observations, J. Geophys. Res., 72, 171-183, 1967.

Sonnerup, B. U. O. and Scheible, M.: Minimum and Maximum Variance Analysis, in: Analysis Methods for Multi-Spacecraft Data, edited by: Paschmann, G. and Daly, P. W., pp. 185-220, ISSI, 1998.

Speiser, T. W. and Ness, N. F.: The Neutral Sheet in the Geomagnetic Tail: Its Motion, Equivalent Currents, and Field Line Connection through It, J. Geophys. Res., 72, 131-141, 1967.

Stone, E. C., Frandsen, A. M., Mewaldt, R. A., Christian, E. R., Margolies, D., Ormes, J. F., and Snow, F.: The Advanced Composition Explorer, Space Sci. Rev., 86, 1-22, doi:10.1023/A: $1005082526237,1998$.

Tsyganenko, N. A.: A magnetospheric magnetic field model with a warped tail current sheet, Planetary and Space Science, 37, 5-20, doi:10.1016/0032-0633(89)90066-4, 1989.

Tsyganenko, N. A., Singer, H. J., and Kasper, J. C.: Storm-time 
distortion of the inner magnetosphere: How severe can it get?, J. Geophys. Res., 108, 1209, doi:10.1029/2002JA009808, 2003.

Viljanen, A. and Häkkinen, L.: Image magnetometer network, in: ESA SP-1198: Satellite-Ground Based Coordination Sourcebook, edited by: Lockwood, M., Wild, M. N., and Opgenoorth, H. J., pp. 111-118, 1997.

Volwerk, M., Glassmeier, K.-H., Runov, A., Nakamura, R., Baumjohann, W., Klecker, B., Richter, I., Balogh, A., Rème, H., and Yumoto, K.: Flow burst-induced large-scale plasma sheet oscillation, J. Geophys. Res., 109, A11208, doi:10.1029/ 2004JA010533, 2004.
Zhang, T. L., Baumjohann, W., Nakamura, R., Balogh, A., and Glassmeier, K.-H.: A wavy twisted neutral sheet observed by CLUSTER, Geophys. Res. Lett., 29(19), L01899, doi:10.1029/2002GL015544, 2002.

Zhang, T. L., Nakamura, R., Volwerk, M., Runov, A., Baumjohann, W., Eichelberger, H. U., Carr, C., Balogh, A., Sergeev, V., Shi, J. K., and Fornacon, K.-H.: Double Star/Cluster observation of neutral sheet oscillations on 5 August 2004, Ann. Geophys., 23, 2909-2914, 2005, http://www.ann-geophys.net/23/2909/2005/. 